\title{
A volume integral implementation of the Goldstein generalised acoustic analogy for unsteady flow simulations
}

\author{
Vasily A. Semiletov, Sergey A. Karabasov \\ School of Engineering and Materials Science, Queen Mary University of London
}

\begin{abstract}
A new volume integral method based on the Goldstein generalised acoustic analogy is developed and directly applied with Large Eddy Simulation (LES). In comparison with the existing Goldstein generalised acoustic analogy implementations the current method does not require the computation and recording of the expensive fluctuating stress auto-covariance function in the seven-dimensional space-time. Until now, the multi-dimensional complexity of the generalised acoustic analogy source term has been the main barrier for using it in routine engineering calculations. The new method only requires local point-wise stresses as an input that can be routinely computed during the flow simulation. On the other hand, the new method is mathematically equivalent to the original Goldstein acoustic analogy formulation and, thus, allows for a direct correspondence between different effective noise sources in the jet and the far-field noise spectra. The implementation is performed for conditions of a high-speed subsonic isothermal jet corresponding to the Rolls-Royce SILOET experiment and uses the LES solution based on the CABARET solver. The flow and noise solutions are validated by comparison with experiment. The accuracy and robustness of the integral volume implementation of the generalised acoustic analogy are compared with those based on the standard Ffowcs Williams -Hawkings surface integral method and the conventional Lighthill acoustic analogy. As a demonstration of its capabilities to investigate
\end{abstract}


jet noise mechanisms, the new integral volume method is applied to analyse the relative importance of various noise generation and propagation components within the Goldstein generalised acoustic analogy model.

Keywords: sound generation by turbulence, jet noise, sound acoustic analogy, Goldstein generalised acoustic analogy, Large Eddy Simulation, CABARET scheme

\section{Introduction}

The theoretical study of jet noise was pioneered by Lighthill (1952) who proposed the first acoustic analogy model. The Lighthill model is as an exact rearrangement of the NavierStokes equations into a linear wave propagation operator and a nominal source term. All the terms of the Navier- Stokes equations not included in the wave operator are grouped together in the source term and expressed as the gradient of the Lighthill stress tensor. The latter tensor includes both the non-linear velocity stress, $\rho v_{i}^{\prime} v_{j}^{\prime}$, which gives rise to the quadrupole-type source and the linear terms, $\rho \bar{v}_{i} v_{j}^{\prime}+\rho \bar{v}_{j} v_{i}^{\prime}$, and $\left(p^{\prime}-c_{\infty}^{2} \rho^{\prime}\right) \delta_{i j}$, the socalled "shear-noise" and "entropy noise" terms, respectively. Here the standard notations of time averaged field and fluctuations with overbar and prime, respectively, are used; $c_{\infty}$ is the sound speed in the free stream, $v_{i}$ is the velocity component in the i-direction of the Cartesian coordinate basis $i, j=1,2,3, \rho$ is density, and $p$ is pressure. The presence of the linear terms, which contain linear meanflow sound propagation and temperature effects, leads to differences in Mach number scaling compared to the sound generated by the nonlinear quadrupole term alone that corresponds to the celebrated Lighthill's $v^{8}$ law for the far-field sound power (Viswanathan 2009, Semiletov and Karabasov 2017).

Since Lighthill, major developments of the acoustic analogy method include Curle (1955) who considered the effect of solid surfaces, Lilley (1958) who took into account meanflow 
sound refraction effects, and Ffowcs Williams (1963) who correctly accounted for the effect of moving sound sources. Goldstein (Goldstein 2002, 2003) proposed the generalised acoustic analogy to account for meanflow propagation effects by exactly re-arranging the governing Navier-Stokes equations into a linear hyperbolic part and the nonlinear acoustic source term which only involves nonlinear stresses, which are expressed in terms of a fluctuation and have zero mean that is a significant improvement over the previous acoustic analogy models.

Early work on idealised flow problems (e.g. Samanta et al. 2006) showed that the Goldstein generalised acoustic analogy has a great promise for the use in unsteady flow simulations as it has the lowest sensitivity to source errors compared to the classical Lighthill's or Lilley's models. However, until present, main applications of the generalised analogy has been limited to the class of 'low-order' noise prediction schemes that use various approximate models to represent the acoustic source strengths (e.g. Goldstein and Leib 2008, Leib and Goldstein 2011, Goldstein 2011, Goldstein at al. 2012) which approximations were 'informed' by Large Eddy Simulation (LES) or Direct Numerical Simulation (DNS) in other works (e.g. Karabasov at al. 2010, Karabasov at al. 2013, Karabasov and Sandberg 2015, Depuru Mohan at al. 2015).

The often use of approximations can be explained by the fact that the Goldstein generalised acoustic analogy model was originally formulated as an integral relation between the autocovariance of fluid stresses and the far-field sound. This relation requires the computation and storage of the corresponding auto-correlation tensor, such as the fourth-order velocity auto-correlation functions for the isothermal jet case, in a seven-dimensional space-time. The fourth rank auto-correlation tensor corresponding to the source term of the generalised acoustic analogy is built from a stress tensor $e^{\prime \prime}{ }_{\mu j}$ of dimension (4x3), which has 9 different 
components, and the total number of different components of the auto-correlation tensor for asymmetric jet flows is $\sum_{1}^{9} n=45$. For axi-symmetric jets, under some reasonable assumptions about symmetry of the turbulence, the number of different source terms can be reduced to 11 independent components (Afsar at al. 2011), which still is a significant number. The resulting source complexity makes direct evaluation of the entire statistical source from unsteady flow simulations extremely challenging.

On the other hand, unsteady flow simulation methods like LES or hybrid Reynolds Averaged Navier Stokes (RANS)-LES have gained popularity for jet noise predictions (Shur et al. 2005, Shur et al. 2015, Faranosov at al. 2013) when applied with the integral surface formulations such as Kirchhoff or Ffowcs Williams -Hawkings (1969) (e.g. di Francescantonio 1997, Brentner and Farassat 1998, Najafi-Yazdi at al. 2011) due to simplicity and efficiency of the latter formulations. In principle, the availability of the timedependent, three-dimensional flow data at high resolution from unsteady flow simulations allows one to investigate jet noise mechanisms at a new level of detail compared to the current space-time resolution available in the experimental studies (e.g. Bridges 2010, Morris and Zaman 2010). However, the computational models based on surface integral formulations tend to be sensitive to the formulation of the far-field propagation model as well as the location and configuration details of the integration surface. Furthermore, compared to the volume integral methods the integral surface methods offer no insight into the actual noise sources in the jet.

Volume integral methods based on the Lighthill acoustic analogy and unsteady flow simulations have also found use for jet noise modelling. For example, in (Freund 2003, Bogey and Bailly 2010) DNS and LES solutions of high-speed jet flows were used, respectively, to compute the Lighthill stress terms, which were then substituted in the acoustic integral to calculate the far-field pressure and the corresponding far-field sound 
power. The computations involved recording the local stress quantities in the jet volume, which was a considerably simpler task compared to computing the auto-covariance function of the corresponding stresses. However, for sound predictions based on the LES that had to resolve a mixture of sound generation and propagation effects that appear in the definition of the Lighthill stress, large errors in overall sound pressure levels were reported. This revealed the importance of explicitly representing linear meanflow propagation effects in the acoustic analogy equations, which are partly accounted for in the Lilley acoustic analogy and, most consistently, in the Goldstein generalised acoustic analogy (for a detailed discussion of the differences between the two analogies see (Goldstein 2010)).

In (Semiletov and Karabasov 2014, Semiletov at al. 2015) the idea of computing the local stresses inside the jet volume and then reconstructing the far-field pressure and the corresponding sound power was extended to the Goldstein generalised acoustic analogy equations. In comparison with the previous implementations of the generalised acoustic analogy based on recording the fourth-order velocity auto-correlation functions, this approach is straightforward for computational implementation and does not require any simplifying assumptions about the effective source configuration in the multi-dimensional space-time. The current article not only provides a systematic and expanded review of these developments but also presents new results of the effective noise source analysis in accordance with the following plan.

In Section 2, the Goldstein generalised acoustic analogy is briefly reviewed and its implementation as a volume integral method is introduced. In Section 3, details of the SILOET benchmark jet case which is used for validation of the method developed is considered, the LES method is introduced, and the flow and noise solutions are compared with available data in the literature and other reference computations. Section 4 is devoted to 
analysis of sound generation and propagation mechanisms based on the volume integral formulation of the Goldstein generalised acoustic analogy developed.

\section{Goldstein generalised acoustic analogy}

\subsection{Formulation based on the auto-covariance of generalised flow stresses}

Following (Goldstein and Leib 2008), time-averaged, Favre-averaged meanflow, and fluctuation variables are introduced

$$
\rho^{\prime}=\rho-\bar{\rho}, p^{\prime}=p-\bar{p}, h^{\prime}=h-\bar{h}, v_{i}^{\prime}=v_{i}-\bar{v}_{i},
$$

where $\rho, p, v$ and $h$ refers to density, pressure, velocity, and enthalpy, respectively; primes denote the fluctuations, and bar and tilde correspond to time and Favre averaging.

By defining the new dependent variables

$$
\begin{gathered}
p_{e}^{\prime}=p^{\prime}+\frac{\gamma-1}{2}\left(\rho v^{\prime 2}-\bar{\rho} \widetilde{v^{\prime 2}}\right), \\
u_{i}=\rho v_{i}^{\prime}
\end{gathered}
$$

the governing Navier -Stokes equations are exactly re-arranged to

$$
\begin{gathered}
D_{0} \rho^{\prime}+\nabla_{j} u_{j}=0, \\
D_{0} u_{i}+u_{i} \nabla_{j} \tilde{v}_{j}+\nabla_{i} p_{e}^{\prime}-\frac{\rho^{\prime}}{\bar{\rho}} \nabla_{j} \tilde{\theta}_{i j}=\nabla_{j} e_{i j}^{\prime \prime}, \\
D_{0} p_{e}^{\prime}+\nabla_{j} \widetilde{c^{2}} u_{j}+(\gamma-1)\left(p_{e}^{\prime} \nabla_{j} \tilde{v}_{j}-\frac{u_{i}}{\bar{\rho}} \nabla_{j} \tilde{\theta}_{i j}\right)=\nabla_{j} e_{4 j}^{\prime \prime}+(\gamma-1) e_{i j}^{\prime \prime} \nabla_{j} \tilde{v}_{i},
\end{gathered}
$$

where

$$
\nabla_{j}=\frac{\partial \cdot}{\partial y_{j}}, D_{0}=\frac{\partial}{\partial t} \cdot+\nabla_{j}\left(\tilde{v}_{j} \cdot\right)
$$

and 


$$
\begin{gathered}
e^{\prime \prime}{ }_{\mu j}=e^{\prime}{ }_{\mu j}-\overline{e_{\mu j}{ }_{\mu j}}, \tilde{\theta}_{\mu j}=\delta_{\mu j} \bar{p}_{e}-\overline{e_{\mu j}^{\prime}} \\
e^{\prime}{ }_{\mu j}=-\rho v_{\mu}^{\prime} v_{j}^{\prime}+\delta_{\mu j} \frac{\gamma-1}{2}{v^{\prime}}^{2}+\left(\sigma_{\mu j}+(\gamma-1) \delta_{\mu 4} \sigma_{j k} v_{k}^{\prime}\right) \\
v_{4}^{\prime}=(\gamma-1)\left(h^{\prime}+\frac{1}{2} v^{\prime 2}\right)=\left(c^{2}\right)^{\prime}+\frac{\gamma-1}{2} v^{\prime 2}, \sigma^{\prime}{ }_{4 j}=-(\gamma-1) q_{j} \\
i, j=1,2,3 ; \mu=1,2,3,4 .
\end{gathered}
$$

In the above equations, Einstein summation is implied, $\sigma_{i j}$ and $q_{j}$ are the viscous stress and heat flux, respectively, $\widetilde{c^{2}}$ is the square of the mean-flow sound speed, $\tilde{\theta}_{i j}$ is the total mean flow stress tensor, and $e^{\prime \prime}{ }_{\mu j}$ is the generalised fluctuating stress tensor. Note that the latter (4x3) tensor includes both the fluctuating Reynolds stresses and the fluctuating enthalpy vector. This is the same term that appears as a separate source component in other formulations of the generalised acoustic analogy (Goldstein 2002, 2003, 2011).

By introducing the far-field microphone and the jet flow coordinates, $\boldsymbol{x}$ and $\boldsymbol{y}$, respectively, the spectral density of the far-field pressure signal can be expressed as the following acoustic integral

$$
S(\boldsymbol{x}, \omega)=\int_{V_{y}} \int_{V_{\Delta}} \hat{R}_{\mu j k l}(\boldsymbol{y}, \boldsymbol{\Delta}, \omega) \hat{\gamma}_{\mu j}(\boldsymbol{y}, \omega \mid \boldsymbol{x}) \hat{\gamma}_{k l}^{*}(\boldsymbol{y}+\boldsymbol{\Delta}, \omega \mid \boldsymbol{x}) d \boldsymbol{\Delta} d \boldsymbol{y}
$$

of the generalised stress tensor auto-covariance

$$
\hat{R}_{\mu j k l}(\boldsymbol{y}, \boldsymbol{\Delta}, \omega)=\int R_{\mu j k l}(\boldsymbol{y}, \boldsymbol{\Delta}, \tau) e^{-i \omega \tau} d \tau,
$$

where $R_{\mu j k l}(\boldsymbol{y}, \boldsymbol{\Delta}, \tau)=\overline{e^{\prime \prime}{ }_{\mu J}(\boldsymbol{y}, \tau) e^{\prime \prime *}{ }_{k l}(\boldsymbol{y}+\boldsymbol{\Delta}, t+\tau)}$ is the generalised stress tensor autocovariance in the time domain, where the overbar means averaging over time $t, i, j=1,2,3$ and $\mu, k=1,2,3,4$.

It is the time-domain generalised stress tensor auto-covariance that can, in principle, be provided from unsteady flow simulations. However, since it is a $4^{\text {th }}$ rank tensor defined over the entire $6+1$ dimensional space-time domain, computation of this quantity is a challenge. Using the standard Fourier transformation formulae, the same quantity can be related to the frequency domain representation of the stresses: 
$\hat{R}_{i j k l}(\boldsymbol{y}, \boldsymbol{\Delta}, \omega)=\overline{\hat{e}^{\prime \prime}{ }_{l j}(\boldsymbol{y}, \omega) \hat{e}^{\prime \prime}{ }_{l j}^{*}(\boldsymbol{y}+\boldsymbol{\Delta}, \omega)}$, where $\hat{e}_{i j}(\boldsymbol{y}, \omega)=\int e_{i j}(\boldsymbol{y}, \tau) e^{-i \omega \tau} d \tau$.

The other part of the far-field sound integral (6) is the propagator operator

$$
\begin{gathered}
\hat{\gamma}_{i j}(\boldsymbol{y}, \omega \mid \boldsymbol{x})=\nabla_{i} \hat{u}_{j}^{a}(\boldsymbol{y}, \omega \mid \boldsymbol{x})+\hat{p}^{a}(\boldsymbol{y}, \omega \mid \boldsymbol{x}) \nabla_{j} \tilde{v}_{j}(\boldsymbol{y}) \\
\hat{\gamma}_{4 j}(\boldsymbol{y}, \omega \mid \boldsymbol{x})=-\nabla_{j} \hat{p}^{a}(\boldsymbol{y}, \omega \mid \boldsymbol{x})
\end{gathered}
$$

that depends on the corresponding adjoint vector Green's function, $\left(\widehat{\boldsymbol{u}}^{a}(\boldsymbol{y}, \omega \mid \boldsymbol{x}), \hat{p}^{a}(\boldsymbol{y}, \omega \mid \boldsymbol{x})\right)$. The adjoint vector Green's function is directly related to the direct tensor Green's function in accordance with the reciprocal theorem between the solution of the direct sound radiation and the adjoint sound scattering problem (e.g. see Tam and Auriault 1998, Karabasov 2010). Compared to solving the direct sound radiation problem, solving the adjoint problem for a small number of far-field microphone locations relative to the number of sources points is computationally advantageous. In the frequency domain, the latter is determined by the following adjoint linearised Euler equations:

$$
\begin{gathered}
D_{1} \hat{\rho}^{a}+\frac{\hat{u}_{i}^{a}}{\bar{\rho}} \nabla_{j} \tilde{\theta}_{i j}=0, \\
D_{1} \hat{u}_{i}^{a}+\nabla_{i} \hat{\rho}^{a}-\hat{u}_{i}^{a} \nabla_{j} \tilde{v}_{j}+\widetilde{c^{2}} \nabla_{j} \hat{p}^{a}+\hat{p}^{a} \frac{\gamma-1}{\bar{\rho}} \nabla_{j} \tilde{\theta}_{i j}=0, \\
D_{1} \hat{p}^{a}+\nabla_{j} \hat{u}_{j}^{a}-(\gamma-1) \hat{p}^{a} \nabla_{j} \tilde{v}_{j}=\delta(\boldsymbol{y}-\boldsymbol{x}),
\end{gathered}
$$

where

$$
D_{1}=i \omega \cdot+\tilde{v}_{j} \nabla_{j}(\cdot)
$$

The solution of these equations can be found by numerically solving the above equations with the delta function source term replaced by an appropriate open-domain radiation boundary condition corresponding to a sink in the far-field observer location (Karabasov and Hynes 2006). The current work, following (Tam and Auriault 1998, Semiletov at al. 2015) uses a simplified semi-analytical locally parallel flow approximation to solve the sound meanflow propagation problem. Briefly, under the locally parallel flow approximation, the jet flow is divided into a series of non-overlapping sections along the jet stream-wise coordinate. Each 
of the sections is stream-wise averaged to correspond to a piece-wise constant flow field in terms of the stream-wise coordinate that becomes a function of radius only, i.e. $\widetilde{\boldsymbol{v}}=$ $(\tilde{v}(r), 0,0), \tilde{c}=\tilde{c}(r)$ of the cylindrical-polar coordinate basis in the jet $\boldsymbol{y}=(r, \theta, z)$. For each jet section, periodic boundary conditions are assumed in the stream-wise z-direction. After such simplifications, the linearised Euler equations can be decomposed into equations for each azimuthal mode separately. This leads to an ordinary differential equation of Rayleigh type for the amplitude of each mode as a function of radius to be solved numerically. Overall, the solution for the amplitudes will be a function of both radius and axial location because of the local meanflow velocity profile used.

It can be remarked that in a number of previous publications in the literature (Goldstein and Leib 2016, Afsar at al. 2016), including some earlier work of the authors (Karabasov et al. 2010, Karabasov at al. 2013), the importance of a fully evolving flow/ sound propagation model for far-field jet noise predictions was stressed. While the effect of a more accurate definition of the Green's function with taking jet flow evolution effects into account compared to the locally parallel flow model will be a subject of future investigations, the present work is the first one in the literature where the effective source term is evaluated directly from LES, avoiding the approximations of previous models. Hence, it is possible that the far-field acoustic solutions, which were obtained in the earlier works as a convolution product of the Green's function propagator with an approximate model of the sevendimensional space-time source term, could have a larger sensitivity to the evolving jet meanflow details compared to the present approach which is approximation-free. 


\subsection{Formulation based on the volume integral approach}

The same far-field power spectral density (6) can be expressed as a complex conjugate of the far-field pressure

$$
S(\boldsymbol{x}, \omega)=\overline{\hat{p}(\boldsymbol{x}, \omega) \hat{p}^{*}(\boldsymbol{x}, \omega)}
$$

where $\hat{p}(\boldsymbol{x}, \omega)$ is the frequency-domain pressure signal at the far-field microphone location

and the overbar indicates statistical ensemble averaging.

In turn, the frequency domain pressure signal can be expressed as a volume integral of the source and the vector adjoint Green's function that satisfies (10),(11). After some rearrangement involving a few integrations by parts, this leads to:

$$
\begin{gathered}
\hat{p}(\boldsymbol{x}, \omega)=\int_{V_{\boldsymbol{y}}}\left\{\hat{e}^{\prime \prime}{ }_{i j}(\boldsymbol{y}, \omega) \nabla_{i} \hat{u}_{j}^{a}(\boldsymbol{y}, \omega \mid \boldsymbol{x})\right. \\
\left.-\frac{i \omega}{\bar{c}^{2}}\left(\hat{e}^{\prime \prime}{ }_{4 j}(\boldsymbol{y}, \omega) \nabla_{j} \hat{p}^{a}(\boldsymbol{y}, \omega \mid \boldsymbol{x})-(\gamma-1) \hat{e}^{\prime \prime}{ }_{i j}(\boldsymbol{y}, \omega) \nabla_{j} \tilde{v}_{i}(\boldsymbol{y}) \hat{p}^{a}(\boldsymbol{y}, \omega \mid \boldsymbol{x})\right)\right\} d \boldsymbol{y} \\
i, j=1,2,3 .
\end{gathered}
$$

Let us assume that $\boldsymbol{e}_{1}$ is the Cartesian coordinate in the stream-wise (jet flow) direction, $\left(\boldsymbol{e}_{2}, \boldsymbol{e}_{3}\right)$ are in the transverse plane, and $\left(\boldsymbol{e}_{1}, \boldsymbol{e}_{2}\right)$ are in-plane with the far-field microphone.

There are three types of noise sources which can be identified in (13): (i) the fluctuating Reynolds stress $\hat{e}^{\prime \prime}{ }_{i j}(\boldsymbol{y}, \omega)$, (ii) the fluctuating stagnation enthalpy stress $\frac{\omega}{\bar{c}^{2}} \hat{e}^{\prime \prime}{ }_{4 j}(\boldsymbol{y}, \omega)$, and (iii) a term associated with the non-divergent meanflow velocity field $\frac{\omega(\gamma-1)}{\bar{c}^{2}} \hat{e}^{\prime \prime}{ }_{i j}(\boldsymbol{y}, \omega) \nabla_{j} \tilde{v}_{i}(\boldsymbol{y})$

For an axi-symmetric meanflow, the 3D integral can be further decomposed into a series of two-dimensional azimuthal modes 


$$
\begin{aligned}
& \hat{p}(\boldsymbol{x}, \omega)=\sum_{n} \hat{p}^{(n)}(\boldsymbol{x}, \omega)=\sum_{n} \int_{V_{y_{\eta}}}\left\{\hat{e}_{i j}^{\prime \prime(n)}\left(\boldsymbol{y}_{\boldsymbol{\eta}}, \omega\right) \nabla_{i} \hat{u}_{j}^{a(n)}\left(\boldsymbol{y}_{\boldsymbol{\eta}}, \omega \mid \boldsymbol{x}\right)-\right. \\
& \left.\hat{e}_{4 j}^{\prime \prime}{ }_{4 j}^{(n)}\left(\boldsymbol{y}_{\boldsymbol{\eta}}, \omega\right) \nabla_{j} \hat{p}^{a(n)}\left(\boldsymbol{y}_{\boldsymbol{\eta}}, \omega \mid \boldsymbol{x}\right)-(\gamma-1) \hat{e}^{\prime \prime}{ }_{i j}^{(n)}\left(\boldsymbol{y}_{\boldsymbol{\eta}}, \omega\right) \nabla_{j} \tilde{v}_{i}\left(\boldsymbol{y}_{\boldsymbol{\eta}}\right) \hat{p}^{a(n)}\left(\boldsymbol{y}_{\boldsymbol{\eta}}, \omega \mid \boldsymbol{x}\right)\right\} \mathbf{d} \boldsymbol{y}_{\boldsymbol{\eta}}
\end{aligned}
$$

using the standard definition of the Fourier transform in the azimuthal direction

$$
f^{(n)}\left(\boldsymbol{y}_{\boldsymbol{\eta}}\right)=\int_{0}^{2 \pi} e^{i n \theta} f(z, r, \theta) \mathrm{d} \theta
$$

Here, $\left(\boldsymbol{y}_{\eta}, \theta\right)=(r, z, \theta)$ is a local cylindrical-polar coordinate basis in the jet, where $r$ and $\theta$ are the radial and azimuthal angle components and $z$ coincides with the jet axis. Vectors with subscript $(n)$ correspond to the $(r, z)$ components of the cylindrical-polar system. The modal representation is useful for reducing the computer storage overheads when recording the stresses in the 3D jet volume since for axi-symmetric jets it is only a relatively small number of modes (compared to the points of the LES grid) which can contribute efficiently to the acoustic integral.

Apparently, the method of computing the far-field spectra density based on the $3 \mathrm{D}$ volume integral formulation (12)-(14) is mathematically identical to the standard formulation (6)-(9). However, there is no calculation of the generalised stress tensor auto-covariance involved in the former volume integral formulation, which leads to a considerable saving of computer memory resources compared to the standard approach. Table 1 compares the computer memory costs for recording the fluctuating stresses required for the volume integral formulation (6)-(9) with those of computing the generalised auto-covariance stress tensor for formulation (12)-(14). The figures are based on the jet case example to be considered in section 3. For simplicity, all numbers are normalised by the computational cost of the volume integral approach. Note that even for a limited number of the tensor components the computation of the auto-covariance function is 5 orders of magnitude more expensive than with the integral volume approach. 
Table 1. Computer memory costs of different implementations of the Goldstein generalised acoustic analogy.

\begin{tabular}{|c|c|c|c|}
\hline & $\begin{array}{l}\text { Volume integral } \\
\text { approach (6)-(9), all } \\
\text { terms }\end{array}$ & $\begin{array}{l}\text { Stress tensor auto-coherence } \\
\text { approach (12)-(14), } 10 \text { auto- } \\
\text { correlation } \\
\text { components }\end{array}$ & $\begin{array}{l}\text { Stress tensor auto- } \\
\text { coherence approach (12)- } \\
\text { (14), all } 45 \text { auto-correlation } \\
\text { tensor components }\end{array}$ \\
\hline Cost & 1 & 328,200 & $1,476,900$ \\
\hline
\end{tabular}

The total computer storage required with the current implementation of the volume integral method for the jet case considered is around $4 \mathrm{~TB}$, which figure could be reduced by performing all pre-processing operations on the fly.

The implementation of the volume integral approach involves the following steps (fig.1):

1. Perform an LES simulation,

2. Calculate all 12 components of the fluctuating stress tensor, $e^{\prime \prime}{ }_{\mu j}$ in the jet volume by applying (5),

3. Fourier transform the stress components in time and in the azimuthal direction, record the several discrete frequencies and modes needed; for the processing of the LES time signals use the signal processing theory [62]: (i) break each fluctuating stress signal into several subintervals with $50 \%$ overlap, (ii) Fourier transform each set, and (iii) record them as several independent frequency-domain realisations of the stresses,

5. Calculate the mean jet flow properties,

6. For each frequency and mode required, calculate the adjoint Green's function, $\widehat{\boldsymbol{u}}^{a(n)}\left(\boldsymbol{y}_{\boldsymbol{\eta}}, \omega \mid \boldsymbol{x}\right), \hat{p}^{a(n)}\left(\boldsymbol{y}_{\boldsymbol{\eta}}, \omega \mid \boldsymbol{x}\right)$, 
7. For each set of the frequency-domain stress fields recorded, integrate the source over the 2D volume, $V_{y_{\eta}}$ and sum over all modes to obtain the far-field pressure signal (14) for each realisation,

8. Calculate the power spectral density by averaging over all realisations of the frequencydomain stress fields available (12).

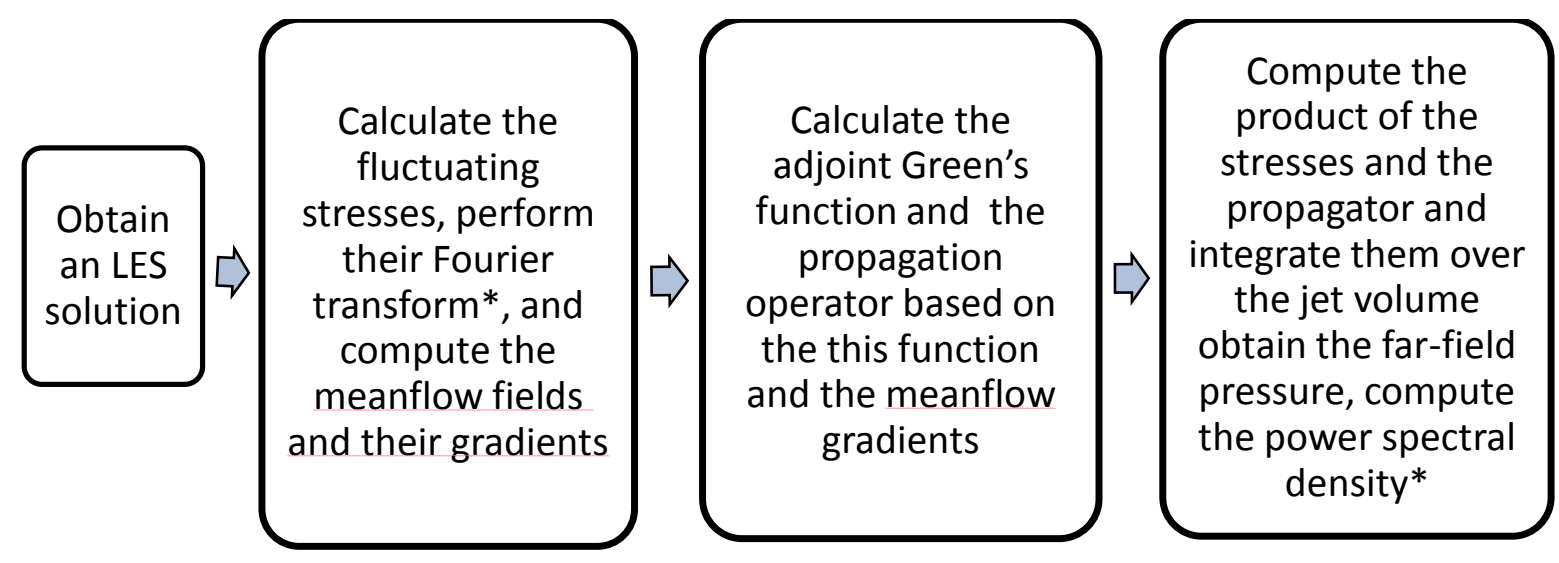

Fig. 1 Flow diagram of the volume integral method based on the generalised acoustic analogy, * indicates the use of signal processing techniques to compensate for a finite duration of the LES time signal.

\subsection{Volume integral formulation and effective source decomposition}

Compared to the standard generalised acoustic analogy approach (12)-(14), which links the effective sources in the jet to the far field sound power, the same direct correspondence is not explicit for the current volume integral formulation. Indeed, the current approach first relates the fluctuating stress terms in the jet to the far-field pressure and then relates the far field pressure to the far-field sound.

To demonstrate how the analysis of far-field noise sources in the jet is also possible within the suggested volume integral approach, let us first decompose the far-field pressure integral 
into contribution of individual modes and the corresponding modes of the vector adjoint Green's function terms using (14). For each mode, the far-field pressure integral can be further broken down into the contributions of different source components

$$
\begin{gathered}
\hat{p}_{e_{i j}}^{(n)}(\boldsymbol{x}, \omega)=\int_{V_{\boldsymbol{y}}} \hat{e}^{\prime \prime}{ }_{i j}^{(n)}\left(\boldsymbol{y}_{\boldsymbol{\eta}}, \omega\right) \nabla_{i} \hat{u}_{j}^{a(n)}\left(\boldsymbol{y}_{\boldsymbol{\eta}}, \omega \mid \boldsymbol{x}\right) \mathbf{d} \boldsymbol{y}_{\boldsymbol{\eta}}, \\
\hat{p}_{e_{4 j}}^{(n)}(\boldsymbol{x}, \omega)=\int_{V_{\boldsymbol{y}}} \hat{e}^{\prime \prime}{ }_{4 j}^{(n)}\left(\boldsymbol{y}_{\boldsymbol{\eta}}, \omega\right) \nabla_{j} \hat{p}^{a(n)}\left(\boldsymbol{y}_{\boldsymbol{\eta}}, \omega \mid \boldsymbol{x}\right) \mathbf{d} \boldsymbol{y}_{\boldsymbol{\eta}}, \\
\hat{p}_{\nabla_{j} \tilde{v}_{i}}^{(n)}(\boldsymbol{x}, \omega)=\int_{V_{\boldsymbol{y}}}(\gamma-1) \hat{e}_{i j}^{\prime \prime}{ }_{i j}^{(n)}\left(\boldsymbol{y}_{\boldsymbol{\eta}}, \omega\right) \nabla_{j} \tilde{v}_{i}\left(\boldsymbol{y}_{\boldsymbol{\eta}}\right) \widehat{p}^{a(n)}\left(\boldsymbol{y}_{\boldsymbol{\eta}}, \omega \mid \boldsymbol{x}\right) \mathbf{d} \boldsymbol{y}_{\boldsymbol{\eta}} .
\end{gathered}
$$

The contributions due to the fluctuating Reynolds stresses can be further decomposed into

$$
\begin{aligned}
& \hat{p}_{e_{11}}^{(n)}(\boldsymbol{x}, \omega)=\int_{V_{y}} \hat{e}^{\prime \prime \prime}{ }_{11}^{(n)}\left(\boldsymbol{y}_{\boldsymbol{\eta}}, \omega\right) \nabla_{1} \hat{u}_{1}^{a(n)}\left(\boldsymbol{y}_{\boldsymbol{\eta}}, \omega \mid \boldsymbol{x}\right) \mathbf{d} \boldsymbol{y}_{\boldsymbol{\eta}}, \\
& \hat{p}_{e_{12}}^{(n)}(\boldsymbol{x}, \omega)=\int_{V_{\boldsymbol{y}}} \hat{e}^{\prime \prime \prime}{ }_{12}^{(n)}\left(\boldsymbol{y}_{\boldsymbol{\eta}}, \omega\right) \nabla_{1} \hat{u}_{2}^{a(n)}\left(\boldsymbol{y}_{\boldsymbol{\eta}}, \omega \mid \boldsymbol{x}\right) \mathbf{d} \boldsymbol{y}_{\boldsymbol{\eta}}, \\
& \hat{p}_{e_{22}}^{(n)}(\boldsymbol{x}, \omega)=\int_{V_{\boldsymbol{y}}} \hat{e}_{22}^{\prime \prime(n)}\left(\boldsymbol{y}_{\boldsymbol{\eta}}, \omega\right) \nabla_{2} \hat{u}_{2}^{a(n)}\left(\boldsymbol{y}_{\boldsymbol{\eta}}, \omega \mid \boldsymbol{x}\right) \mathbf{d} \boldsymbol{y}_{\boldsymbol{\eta}}, \text { and so on. }
\end{aligned}
$$

By disabling all source terms except from any user defined stress and mode component of the pressure integral $\hat{p}(\boldsymbol{x}, \omega)$, the contribution of this particular source term in the far-field power spectra $\hat{P}(\boldsymbol{x}, \omega)$ can be calculated.

Furthermore, if the effect of cross-products in the power spectra integral can be ignored, that is, only symmetric terms of the generalised stress tensor auto-covariance, $R_{\mu j \mu j}$ are important for the far-field sound. The source symmetry property is consistent with observations made for other high-speed axi-symmetric jets (e.g. comp. with $R_{1111}, R_{2222}, R_{3333}, R_{1212}, R_{1313}$, and $R_{2323}$ main source components considered in (Karabasov at al. 2010)), the sound power spectra can be decomposed into the contributions of individual terms: 


$$
\begin{gathered}
\hat{P}(\boldsymbol{x}, \omega)=\sum_{n, m} \overline{\hat{p}^{(n)}(\boldsymbol{x}, \omega) \hat{p}^{(m) *}(\boldsymbol{x}, \omega)} \approx \sum_{n} \overline{\hat{p}^{(n)}(\boldsymbol{x}, \omega) \hat{p}^{(n) *}(\boldsymbol{x}, \omega)} \approx \\
\sum_{n} \overline{\hat{p}_{e_{4 j}}^{(n)}(\boldsymbol{x}, \omega) \hat{p}_{e_{4 j}}^{(n) *}(\boldsymbol{x}, \omega)}+\sum_{n} \overline{\hat{p}_{\nabla_{j} \tilde{v}_{l}}^{(n)}(\boldsymbol{x}, \omega) \hat{p}_{\nabla_{j} \tilde{v}_{l}}^{(n) *}(\boldsymbol{x}, \omega)}+\sum_{n} \overline{\hat{p}_{e_{l \jmath}}^{(n)}(\boldsymbol{x}, \omega) \hat{p}_{e_{l \jmath}}^{(n) *}(\boldsymbol{x}, \omega)}= \\
\sum_{n} \overline{\hat{p}_{e_{4 j}}^{(n)}(\boldsymbol{x}, \omega) \hat{p}_{e_{4 j}}^{(n) *}(\boldsymbol{x}, \omega)}+\sum_{n} \overline{\hat{p}_{\nabla_{j} \tilde{v}_{l}}^{(n)}(\boldsymbol{x}, \omega) \hat{p}_{\nabla_{\jmath} \tilde{v}_{l}}^{(n) *}(\boldsymbol{x}, \omega)}+\sum_{n} \overline{\hat{p}_{e_{11}}^{(n)}(\boldsymbol{x}, \omega) \hat{p}_{e_{11}}^{(n) *}(\boldsymbol{x}, \omega)}+ \\
\sum_{n} \overline{\hat{p}_{e_{12}}^{(n)}(\boldsymbol{x}, \omega) \hat{p}_{e_{12}}^{(n) *}(\boldsymbol{x}, \omega)}+\sum_{n} \overline{\hat{p}_{e_{21}}^{(n)}(\boldsymbol{x}, \omega) \hat{p}_{e_{21}}^{(n) *}(\boldsymbol{x}, \omega)}+\cdots,
\end{gathered}
$$

The former property can be referred to as mutual orthogonality of the individual source components with respect to the far-field sound power. Note that it does not only require the symmetry of the autocorrelation source tensor but also the orthogonality of the source modes for the far-field noise solution, which was also previously discussed in jet noise literature (Goldstein and Leib 2016). In section 4, it will be demonstrated how both these conditions are satisfied for the SILOET jet case.

Besides, if the density distribution of any particular source component corresponding to a given stress term needs to be analysed in jet volume, this can also be achieved by noting that, for example, that

$$
P_{e_{i j}}^{(n)}=\overline{\hat{p}_{e_{l j}}^{(n)}(\boldsymbol{x}, \omega) \hat{p}_{e_{l j}}^{(n) *}(\boldsymbol{x}, \omega)}=
$$

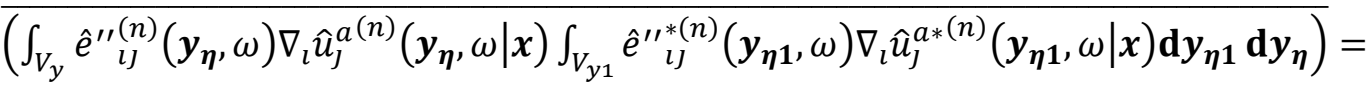

$$
\begin{aligned}
& \int_{V_{y}} \widetilde{P}_{e_{i j}}^{(n)}\left(\boldsymbol{y}_{\boldsymbol{\eta}}\right) \mathbf{d} \boldsymbol{y}_{\boldsymbol{\eta}},
\end{aligned}
$$

where $\tilde{P}_{e_{i j}}^{(n)}\left(\boldsymbol{y}_{\boldsymbol{\eta}}\right)$ is the corresponding source density function given by

$$
\tilde{P}_{e_{i j}}^{(n)}\left(\boldsymbol{y}_{\boldsymbol{\eta}}\right)=\nabla_{i} \hat{u}_{j}^{a(n)}\left(\boldsymbol{y}_{\boldsymbol{\eta}}, \omega \mid \boldsymbol{x}\right) \overline{\hat{e}^{\prime \prime}{ }_{l j}^{(n)}\left(\boldsymbol{y}_{\boldsymbol{\eta}}, \omega\right)\left(\int_{V_{\boldsymbol{y} 1}}{\hat{e^{\prime}}}_{l j}^{\prime *(n)}\left(\boldsymbol{y}_{\boldsymbol{\eta} \mathbf{1}}, \omega\right) \nabla_{l} \hat{u}_{J}^{a *(n)}\left(\boldsymbol{y}_{\boldsymbol{\eta} \mathbf{1}}, \omega \mid \boldsymbol{x}\right) \mathbf{d} \boldsymbol{y}_{\boldsymbol{\eta} \mathbf{1}}\right)} .
$$

Note also that although, inside the integral, (19) implicitly includes the $4^{\text {th }}$ rank auto covariance tensor, $\hat{R}_{i j i j}^{(n)}\left(\boldsymbol{y}_{\boldsymbol{\eta}}, \boldsymbol{y}_{\boldsymbol{\eta} \mathbf{1}^{1}}, \omega\right)=\overline{\hat{e}_{i j}^{\prime \prime(n)}\left(\boldsymbol{y}_{\boldsymbol{\eta}}, \omega\right) \hat{e}_{i j}^{\prime \prime *(n)}\left(\boldsymbol{y}_{\boldsymbol{\eta} \mathbf{1}}, \omega\right)}$, the latter expensive function 
does not need to be recorded. Indeed, the computation of the effective source density, $\tilde{P}_{e_{i j}}^{(n)}(\mathbf{y})$ only requires the storage of several realisations of turbulent stresses $\hat{e}^{\prime \prime}{ }_{i j}^{(n)}\left(\boldsymbol{y}_{\boldsymbol{\eta}}, \omega\right)$ and the vector adjoint Green's function $\nabla_{i} \hat{u}_{j}^{a}\left(\boldsymbol{y}_{\boldsymbol{\eta}}, \omega \mid \boldsymbol{x}\right)$ for azimuthal modes and frequencies of interest as outlined in section 3.2. $\tilde{P}_{e_{i j}}^{(n)}(\mathbf{y})$ can then be reconstructed in the entire 3D jet volume as the following: (i) calculate the inner integral $\int_{V_{y 1}} \hat{e}_{i j}^{\prime \prime \prime(n)}\left(\boldsymbol{y}_{\boldsymbol{\eta} \mathbf{1}}, \omega\right) \nabla_{i} \hat{u}_{j}^{* a}\left(\boldsymbol{y}_{\boldsymbol{\eta} \mathbf{1}}, \omega \mid \boldsymbol{x}\right) \mathbf{d} \boldsymbol{y}_{\boldsymbol{\eta} \mathbf{1}}$ for each realisation of the stress field, (ii) multiply the result by all possible realisation values of the stress field $\hat{e}_{i j}^{\prime \prime(n)}\left(\boldsymbol{y}_{\boldsymbol{\eta}}, \omega\right)$, and (iii) ensemble average over the combined number of realisations and multiply by the Green's function, $\nabla_{i} \hat{u}_{j}^{a(n)}\left(\boldsymbol{y}_{\boldsymbol{\eta}}, \omega \mid \boldsymbol{x}\right)$.

\section{Validation for the SILOET experiment data}

\subsection{Jet case definition and flow solution validation}

For validation of the new volume integral method developed, jet conditions from the RollsRoyce Strategic Investment in Low-carbon Engine Technology (SILOET) experiment performed in the Noise Test Facility (NTF) of QinetiQ are considered (SILOET Programme Rolls-Royce private data). The conditions correspond to a high-speed single-stream subsonic isothermal jet flow issuing from a convergent profiled nozzle. Details of the jet case are summarised in table 2. Two conditions are considered: a zero coflow (the static jet case) and a coflow velocity $U_{c}$ in the jet stream-wise direction corresponding to $M_{c}=0.3$, where $M_{c}=$ $U_{c} / c_{\infty}$

Table 2. Parameters of the Rolls-Royce SILOET jet case

\begin{tabular}{|l|l|l|l|l|}
\hline Nozzle & Contraction & Acoustic Mach & Reynolds & Temperature \\
diameter, $D_{j}$ & ratio & number, $U_{j} / c_{\infty}$ & number, $U_{j} D_{j} / v$ & ratio, $T_{j} / T_{\infty}$ \\
\hline 0.1016 & 2.56 & 0.875 & $2 \cdot 10^{6}$ & 1 \\
\hline
\end{tabular}


The flow solutions have been obtained with the MILES CABARET solver (Faranosov at al. 2013, Semiletov and Karabasov 2013, 2014) on a hexahedral cylindrical grid of circa $2110^{6}$ cells in total. For unsteady flow modelling, the governing Navier-Stokes equations are solved in the framework of Monotonically Integrated LES (MILES) method (Fureby and Grinstein 2002). The MILES implementation used is based on the low-dissipative and low-dispersive CABARET method (Goloviznin and Samarskii 1998, Karabasov and Goloviznin 2009, Chintagunta at al. 2017) on a hexahedral cylindrical grid of circa $2110^{6}$ cells in total. Numerical simulations have been performed over approximately 560 Time Units (TUs), which includes about 200 TUs of the initial solution spin-out time. Here one TU corresponds to the time required for an eddy to travel over one jet diameter, $\mathrm{TU}=D_{j} / U_{j}$.

The computational domain is covered with a cylindrical-type computational grid with a Cartesian bloc along the centreline. The grid includes the jet flow downstream of the nozzle exit and spans approximately over 100 jet diameters axially and 30 jet diameters radially. For open boundaries, a combination of characteristic non-reflecting boundary conditions and grid stretching is used to minimise spurious reflections. The standard non-slip adiabatic boundary condition is applied on the nozzle walls. Upstream of the nozzle exit, the computational domain includes a part of the nozzle and spreads over 5 jet diameters.

Most of the grid cells are clustered in the shear layer region which is the most important zone for noise generation. Fig. 2 shows details of the grid spacing distribution along the jet lip line and radially. The grid has a sufficient resolution for acoustic wave propagation upto $S t \sim 1-$ 2 over the first 8-15 jet diameters from the nozzle exit, where $S t=f D_{j} / U_{j}$ is the Strouhal number defined by the nozzle diameter. 
At the nozzle lip, the grid cell sizes in the radial, stream-wise, and azimuthal direction are $\Delta r=0.005 D_{j}, \Delta z=0.025 D_{j}, r \Delta \theta=0.0245 D_{j}$, respectively. This resolution is likely to be too coarse to capture the boundary layer at the nozzle exit and, while no boundary layer modelling was attempted since there are no flow measurements available inside the nozzle from the experiment, this resulted in an initially laminar jet condition in the LES solution. Note that using the laminar jet inflow condition, which not only relies on the fast transition of the flow solution to turbulence in accordance with the shear layer instability development downstream of the nozzle exit but also ignores the fact that the jet flow at such high Reynolds number is always turbulent at the nozzle exit. Hence, the current idealised treatment of the jet inflow conditions makes the jet development dependent on the numerical method details, which may not be ideal according to the existing jet noise literature (Bogey at al. 2012, Bres at al. 2015). Obtaining a more accurate LES solution for the same jet case with accounting for a realistic turbulent jet inflow condition, for example, based on the wall modelled LES approach (Bres at al. 2015), will be a subject of the future work. In the meantime, it should be pointed out that similar idealised laminar inflow jet conditions were used in other publications on isolated jets (Bres at al. 2012, Ingraham at al. 2017, Leib at al. 2017) without reporting any drastic effect of the initially laminar condition on the downstream jet flow development that defines the far-field noise spectra and acoustic sensitive jet flow properties such as the fourth-order velocity auto-correlation functions. Most recently, Markesteijn and Karabasov (2018) used the MILES CABARET method for computing the NASA jets corresponding to Set Points 7 and 3 conditions at Reynolds numbers around $10^{6}$ on Graphics Processing Units (GPUs) to reduce the LES run time. The GPU LES solutions obtained with imposing a laminar inflow jet condition were compared with the solutions obtained by introducing a numerical turbulence grid upstream of the nozzle exit to capture the same velocity fluctuations at the nozzle exit as reported in the NASA experiment. In both cases, 
with and without imposing the initial disturbances at the nozzle exit, the obtained LES solutions showed a similar good agreement with the experiment for predicting the rest of the flow field and the far-field noise spectra.

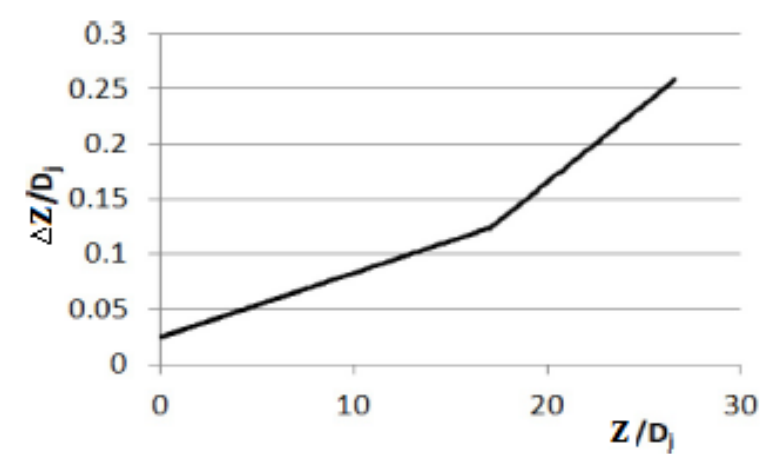

(a)

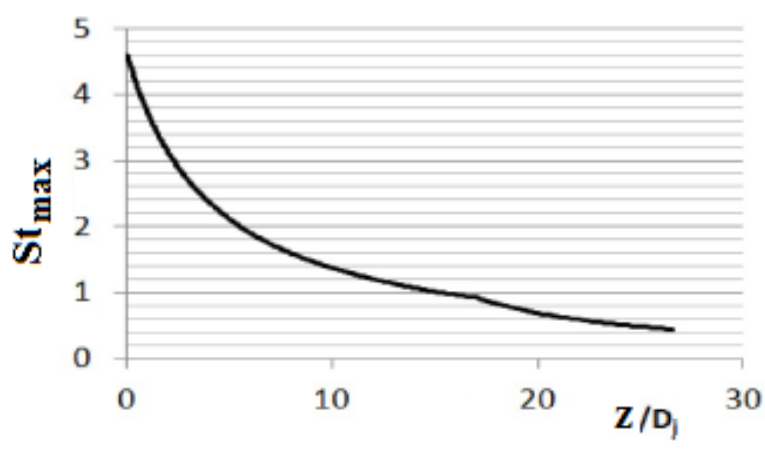

(c)

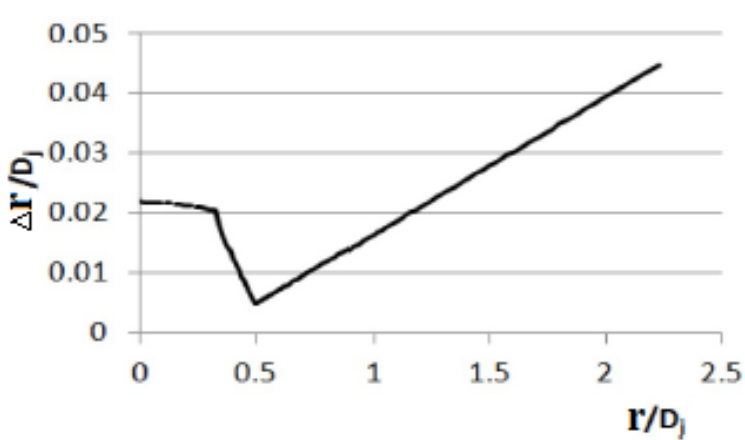

(b)

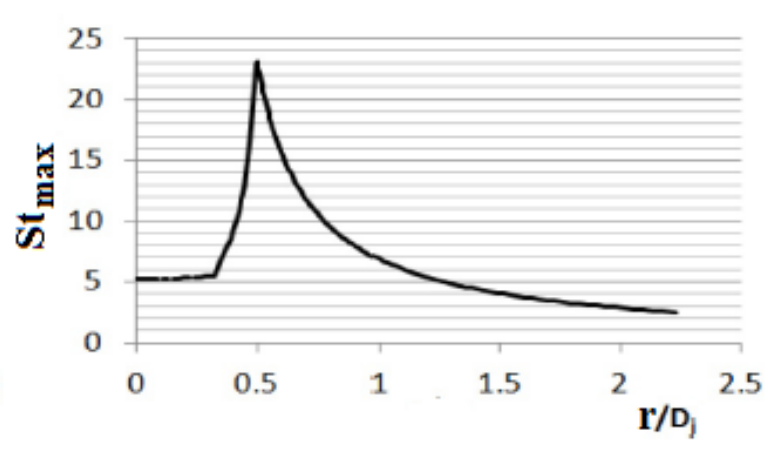

(d)

Fig. 2 Cell size distribution in the axial and radial direction, (a) and (b), and the corresponding highest resolved acoustic frequencies, (c) and (d).

Fig. 3 shows results of the validation of the presented CABARET SILOET flow solutions in comparison with the experimental data available in the literature.

Fig.3a compares the centreline profile of the axial meanflow velocity component with the function, $\frac{\bar{v}_{Z}}{U_{j}}=1-e^{\alpha /\left(1-z / Z_{0}\right)}$, where $Z_{0}$ is the potential core length of the jet and $\alpha=1.43$ is the Witze empirical parameter, which describes a similitude jet profile, as commonly accepted in high-speed jet flow experiments (Witze 1974). 
Fig.3b and c compare the current LES solutions for jet lipline distributions of the stream-wise and the transverse velocity component fluctuations with the experimental data from (Lau at al. 1979, Bridges and Wernet 2003, Bridges 2010) as well as with the Implicit LES solution obtained on a similar resolution grid ( 20 $10^{6}$ grid cells) from (Shur at al. 2015) that corresponds to jet conditions of the Boeing experiment (Viswanathan 2004).

To further analyse the current SILOET LES solution in the noise sensitive shear layer region, following (Bogey and Bailly 2010), the stream-wise profile of the shear-layer momentum thickness

$$
\delta_{\theta}=\int_{0}^{r_{0.04}} \frac{\bar{v}_{Z}}{\bar{U}_{c}}\left(1-\frac{\bar{v}_{Z}}{\bar{U}_{c}}\right) d r
$$

is considered, where $\bar{v}_{z}$ is the local axial meanflow velocity, $\bar{U}_{c}$ is the corresponding axial velocity value at the jet centreline, and $r_{0.04}$ is defined so that $\bar{v}_{z}\left(r_{0.04}\right)=0.04 \bar{U}_{c}$.

Fig.3d compares the above quantity computed from the present LES solution with the experimental data available from (Hussein at al. 1994). In addition, LES solutions from several references digitised from (Bogey and Bailly 2010) are shown on the same plot. In the latter case, the several solutions correspond to various shear layer resolution at the nozzle exit $\left(r / D_{j}=0.005\right.$ and 0.0025 denoted by JetD005 and JetD0025, respectively) and with and without additional boundary layer tripping inside their pipe nozzle (JetD005p2000 and JetD005, respectively). In each case, the normalisation based on the jet radius at the nozzle exit $r_{0}=D_{j} / 2$ is applied. 


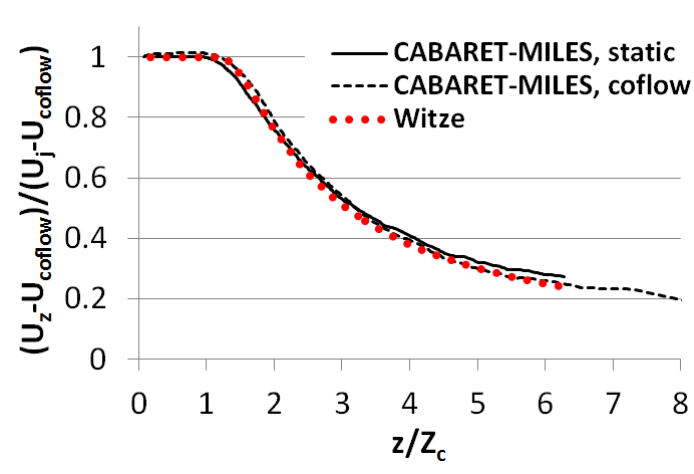

(a)

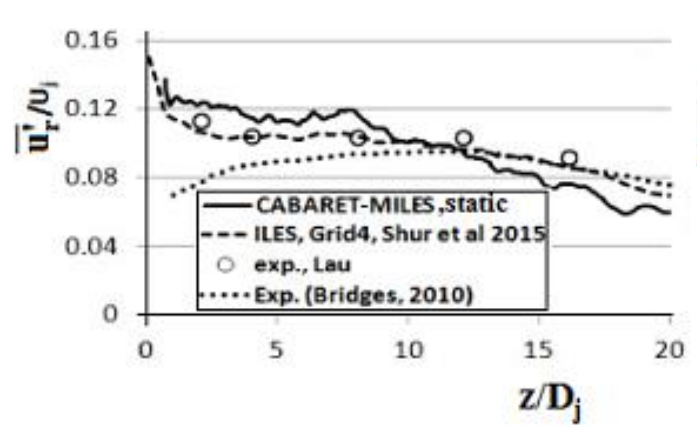

(c)

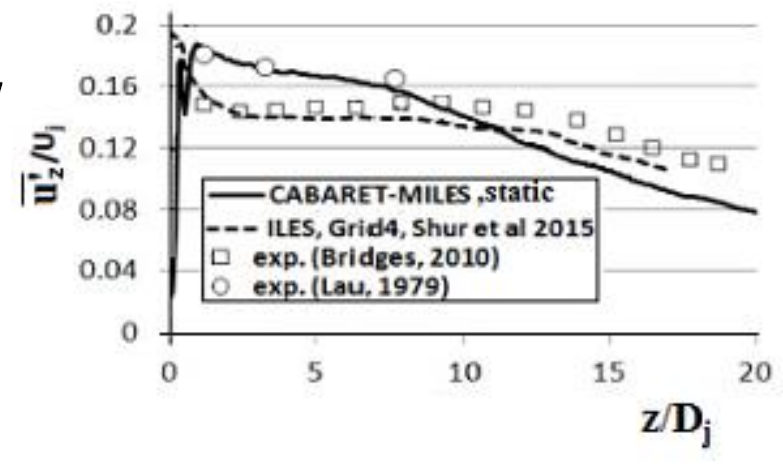

(b)

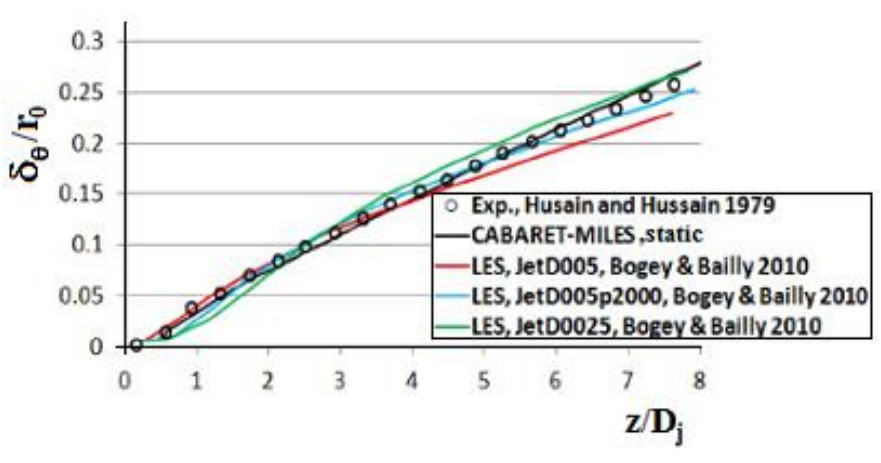

(d)

Fig.3 Comparison of the CABARET SILOET flow solution with the experimental and computational data available: centreline meanflow velocity profile (a), lipline profile of the axial velocity fluctuation (b), lipline profile of the radial velocity fluctuation (c), stream-wise profile of the shear layer thickness (d).

Notably, in all cases the present CABARET SILOET simulation is in a good agreement with the referenced experimental and computational data.

To conclude the LES validation section, the turbulent velocity spectra from the current jet flow solution for a few representative points in the shear layer are extracted and their slope is compared with the $-5 / 3$ that is representative of the inertial range of turbulence cascade in accordance with Kolmogorov's theory (Pope 2000). Because of the limited length of time history available from the LES solution, the spectra are calculated in accordance with the 
standard signal post-processing method (Welch 1967) to improve the statistical convergence. Again, the original signal is divided into several sub-sample signals with 50\%-overlap. Each sub-sample signal is Fourier transformed with the Hanning window applied and the final spectra result is obtained by averaging over all sections. This is the same signal postprocessing technique which is used in the implementation of the volume integral method outlined in sections $2.2,2.3$. The results are presented in fig. 4 which shows that the current LES solution captures the $-5 / 3$ slope over at least one octave of the turbulent velocity spectra as expected from a high Reynolds number flow.

At this point it may be concluded that the laminar inflow jet condition approximation used for the current LES solution did not lead to a significant change in the jet flow dynamics downstream from the nozzle exit in comparison with the existing literature. Therefore, despite the approximation made, the quality of the current LES flow solution is reasonable in terms of its further input in the acoustic analogy modelling that is the core part of the current publication.

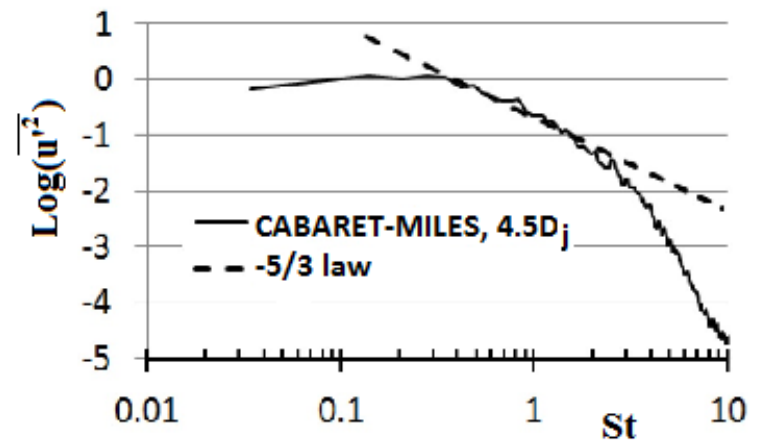

(a)

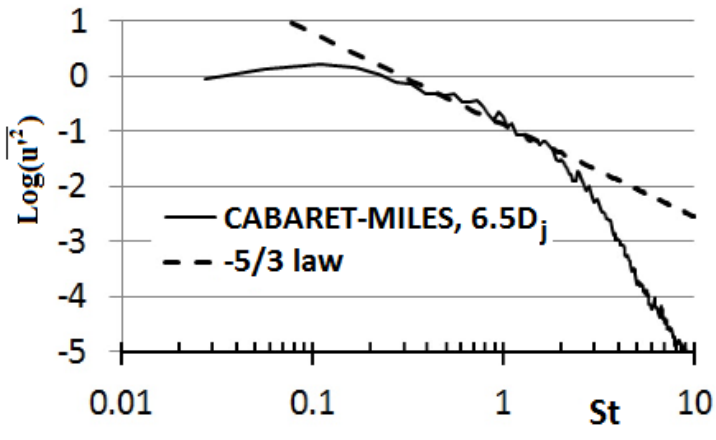

(b)

Fig.4 CABARET SILOET results for the turbulent velocity spectra at two representative jet lipeline locations. 


\subsection{Far-field noise spectra predictions and comparison with the Ffowcs Williams - Hawkings method}

Having selected a sufficiently large cylindrical part of the computational domain $\left(25 D_{j}\right.$ axially times $2 D_{j}$ radially) downstream of the nozzle exit, all components of the generalised fluctuating turbulent stresses $e^{\prime \prime}{ }_{\mu j}$ are extracted from the LES solution and decomposed into 10 azimuthal modes and 50 frequencies within $0.05<S t<3$. The resulting fields stress fields are substituted in the volume integral formulation of the generalised acoustic analogy (12),(14) to compute the far-field noise spectra and compare the result with the far-field microscope measurements from the SILOET experiment. The far-field microphone data correspond to a distance of $120 D_{j}$ from the centre of the nozzle exit.

For statistical averaging of the fluctuating stress signals obtained from LES, there are 20 subintervals with $50 \%$ overlap used. The computational cost of the volume integral solution is mainly due to computing the integral sums for multiple realisations of the LES stress fields required since the computation of the semi-analytical Green's function is extremely fast. The total cost of applying the volume integral method to calculate the far-field noise is a small fraction of the computational cost of the LES solution ( 20 minutes on a workstation compared to a few weeks on a big computer cluster with hundreds of parallel processes, respectively).

In terms of the computer memory, the major cost of the volume integral method is due to recording of the various realisations of the 12 generalised stress components in the $3 \mathrm{D}$ jet volume. However, since the stresses only need recording for a discrete number of frequencies, while the Fourier transformation is performed during the simulation run, and the flow solution is required for a limited number of azimuthal modes, the total cost is only marginally larger compared to that of standard 3D surface integral methods. 
To cross-verify the far-field noise predictions of the volume integral formulation of the Goldstein generalised acoustic analogy, the same LES solution is applied in the framework of the penetrable formulation of the Ffowcs Williams - Hawkings (FW-H) surface integral method. The method is based on computing the acoustic integrands corresponding to the "thickness" and "dipole" terms on a control surface that confines the jet with all important noise sources (di Francescantonio 1997, Brentner and Farassat 1998) and propagating the solution to the far-field using the analytical free-space Green's function. Under the assumption that all significant noise sources are included inside the control surface, the external quadrupole sources are ignored. Following (Shur at al. 2015), a sufficient number of closing discs $(\sim 10)$ at about 25 jet diameters downstream from the nozzle exit are used. An average far-field prediction of those obtained with the same funnel-shape control surface with different closing discs (fig.5) was used to filter out the pseudo-sound effect due to vorticity waves crossing the control surface. Upstream of the nozzle exit, the control surface confines the nozzle lip and is left open. Furthermore, since the solution of the penetrable formulation of the FW-H method is known to be sensitive to the location of integration surfaces, several of them (denoted as "sets" in fig.5) have been considered before the optimum one (Set\#2) was selected to make sure that the acoustic integration surface is located within the region of a sufficient grid resolution while sufficiently far away from the vorticity-rich zones. To process the time signal at the far-field microphone location, the same signal processing method based on the Fourier transform with the sub-sample averaging (Welch 1967) is applied as in the volume integral method based on the acoustic analogy. 


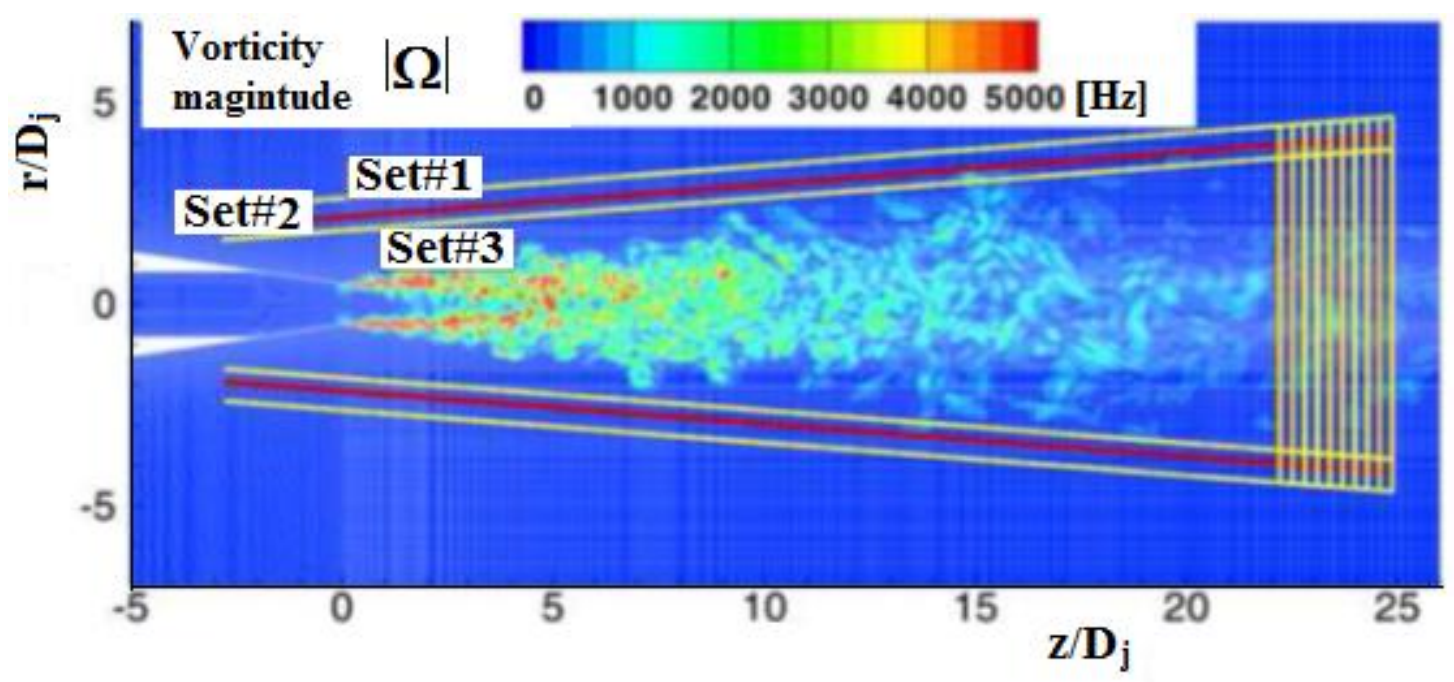

Fig. 5 Location of integration surfaces used with the Ffowcs Williams - Hawkings method relative to a typical distribution of the instantaneous vorticity magnitude in the SILOET jet.

Fig.6 compares sound power spectra density (PSD) obtained with the Goldstein generalised acoustic analogy implementation (12),(14) and with those of the FW-H method. Both predictions are within 2-3 dB from the experiment from $S t \sim 0.1-0.2$ upto $S t \sim 2-3$ for polar angles $30^{\circ}-90^{\circ}$ to the jet axis.

Note that, for the $30^{\circ}$ angle to the jet, the frequencies lower than $S t \sim 0.2$ are attenuated for both of the FW-H and the acoustic analogy solution (resulting in a $\sim 4 \mathrm{~dB}$ error with respect to the experiment). This attenuation is likely due to the insufficient LES grid resolution at the end of the potential core of the SILOET jet. This is also in accordance with the results of Markesteijn and Karabasov (2018) who examined the grid sensitivity of the MILES CABARET solutions with the FW-H method on several LES grids circa 60-80 $10^{6}$ cells with various refinements for similar high-speed jet cases. Besides, for the same $30^{\circ}$ angle to the jet flow, the acoustic analogy solution appears to be less accurate in the medium frequency range, $0.2<S t<0.4$ in comparison with the FW-H prediction that is virtually "spot on" for $30^{0}$ angle, e.g. remains within $0.5-1 \mathrm{~dB}$ accuracy with respect to the experiment for $0.2<S t$ 
<1.5. One explanation for the discrepancy could be the simplified locally parallel flow approximation that was applied for solving the sound meanflow propagation problem in the current acoustic analogy implementation. Hence, future work will be devoted to implementing a more sophisticated evolving meanflow propagation model within the suggested volume integral approach. For example, further steps may involve a numerical solution of the adjoint linearised Euler equations for the fully evolving jet flow following the method of (Karabasov and Hynes, 2006) and using non-parallel flow asymptotics within the semi-analytical approach suggested in (Sassanis et al. 2017). On the other hand, it should also be recalled that, in comparison with the $\mathrm{FW}-\mathrm{H}$ solution, there is no single calibration parameter involved in defining the integration domain of the acoustic analogy method, hence, the current predictions of the acoustic analogy method were not at all "calibrated" compared to the FW-H solution based on the optimal control integration surface. This means that the 0.5-1dB accuracy achieved with the FW-H method for the $30^{\circ}$ angle noise spectrum predictions on the current 21 million LES grid could be slightly fortuitous and future work should also involve improving the LES grid resolution for the current jet case.

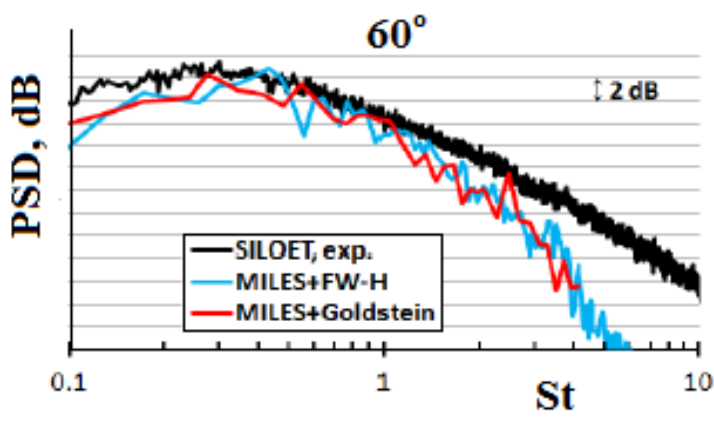

(a)

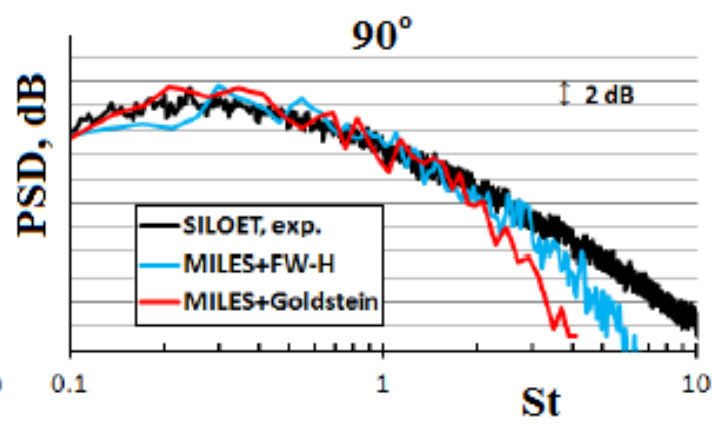

(b) 


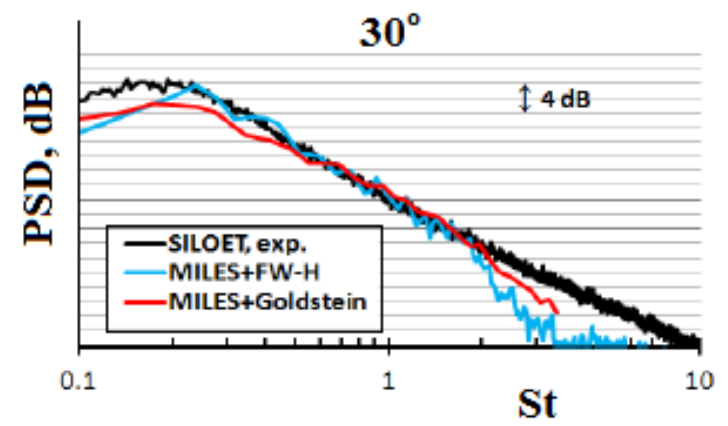

(c)

Fig.6 Validation of the far-field noise spectra predictions of the volume integral acoustic analogy method in comparison with the experiment and the reference FW-H solution for the isothermal static SILOET jet: $90^{\circ}$ polar angle (a), $60^{\circ}$ polar angle (b), and $30^{\circ}$ (c) polar angle to the jet flow.

To demonstrate the importance of the absence of calibration parameters for jet noise predictions, the second SILOET jet case, which corresponds to a coflow in the jet streamwise direction at $M_{c}=0.3$ is considered. Similar to the static jet case, the CABARET solution is first obtained for the SILOET jet flow case with taking the coflow effect into account. The LES solution is then applied for the volume integral formulation as well as for the penetrable FW-H formulation without any additional calibration of either of the acoustic modelling methods. For the FW-H method, this meant using the same acoustic control surface in the coflow case as in the previous static jet case.

Fig.7 compares the sound spectra predictions by both methods and with the experiment for $90^{\circ}$ observer location. There is a strong amplification of the FW-H solution notable at low frequencies, which is a manifestation of spurious pseudosound waves that were not sufficiently abated in this case. Note that there are recipes in the FW-H literature (e.g. Shur at al. 2005), how to adjust the acoustic surface location depending on the jet operating conditions to filter out the pseudo sound effects, but these recipes are empirical in nature. In 
comparison with these, the noise spectra predictions of the current volume integral method based on the acoustic analogy are within 2-3 dB from the experiment both for the static and the coflow jet conditions without any empirical calibration needed.

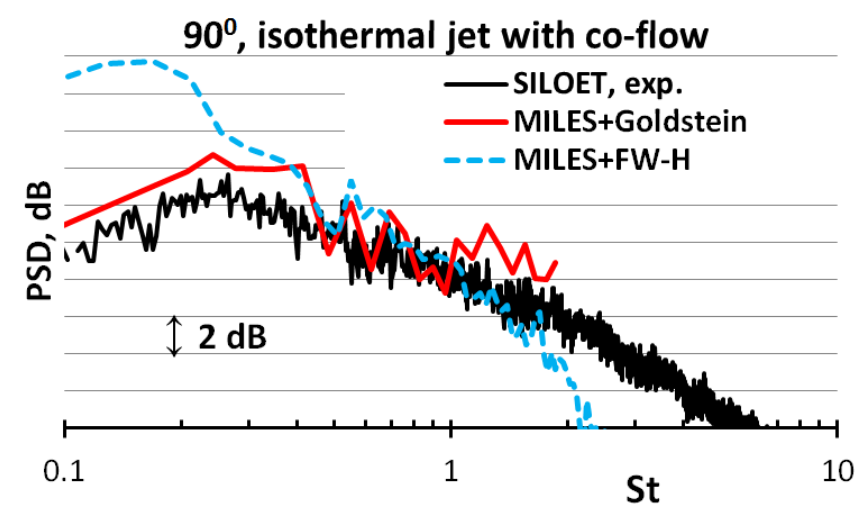

Fig. 7 Far-field noise spectra of the isothermal SILOET jet with the coflow for $90^{\circ}$ polar angle: comparison of the volume integral method predictions with those of the FW-H method and with the experiment.

\section{Noise generation and propagation mechanisms}

Having validated the volume integral method for far-field noise spectra prediction in the previous section, here we will use it to analyse the contribution of individual sound generation and propagation components of the Goldstein generalised acoustic analogy model for the static SILOET jet.

\subsection{Contribution of the source volume to the far-field noise}

Fig.8 shows the far-field spectra predictions based on various sub-domains of the original integration volume for $90^{\circ}$ microphone angle. Each of the small parts of the original control volume has a cylindrical shape and starts at the nozzle exit. The radius and the stream-wise extent of the control volumes are varied one at a time. The change of the far-field sound spectra due to decrease of the integration domain in the stream-wise direction down to the cylindrical volume $(2 \times 4) D_{j}$ (radially times axially) is demonstrated in Fig.8a and the same for the radial direction down to $(0.75 \times 25) D_{j}$ (radially times axially) is shown in Fig.8b. In 
both cases, as the size of the source volume decreases the low frequencies become attenuated whereas the frequencies higher than $S t \sim 0.4$ remain almost unaffected by the control volume change applied. Furthermore, starting from a certain elongated cylinder shape of the source volume, which is approximately $(1.5 \mathrm{x} 10) D_{j}$ (radially times axially) that is about a factor of 2 larger than the jet plume dimensions (the potential core length of the SILOET jet is about 5.5 $D_{j}$ ), the same relative change of the control volume in the radial or the stream-wise direction leads to approximately the same noise spectra. For example, the effect of the change of control volume in the radial direction from $1.5 D_{j}$ to $0.75 D_{j}$ on low part of the frequency spectra is approximately the same as that of the change in the stream-wise direction from 15 $D_{j}$ to $6 D_{j}$. Results of varying the size of the integration volume for the $30^{\circ}$ angle spectra (not shown) demonstrate the same effect. This suggests that the effective source shape, which in terms of the auto-covariance of fluctuating turbulent stresses (6) corresponds to the source correlation volume, changes approximately in proportion with the jet dimensions. The effective source shrinks for high frequency sound and the high-frequency sources tend to be more compactly located closer to the nozzle exit and the jet lipline area compared to the low frequencies.

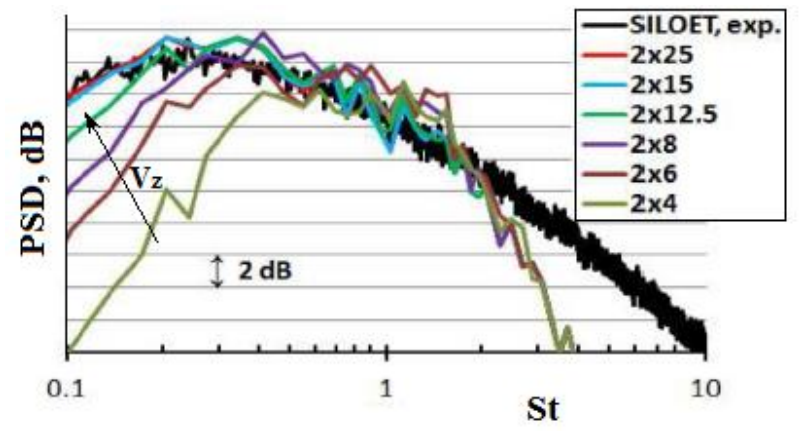

(a)

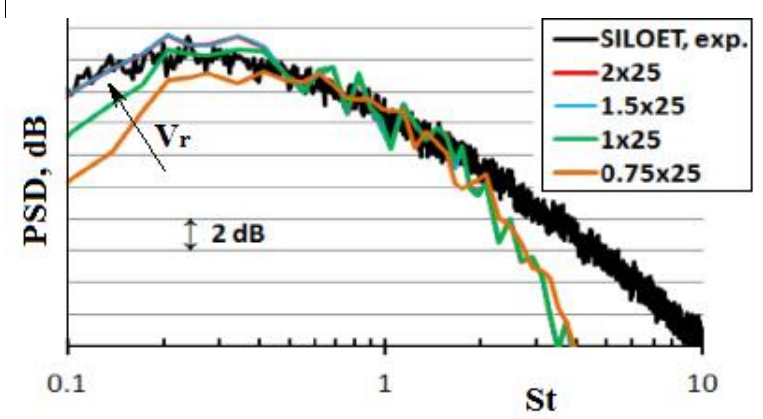

(b)

Fig.8 Effect of shrinking the cylindrical source integration domain on the far-field noise spectra at $90^{\circ}$ observer angle: varying the source volume in the stream-wise direction, $\mathrm{V}_{\mathrm{z}}(\mathrm{a})$ and the radial direction, $\mathrm{V}_{\mathrm{r}}(\mathrm{b})$. 


\subsection{Orthogonality of different source components for far-field power noise spectra}

Fig.9 shows far-field noise spectra predictions for two typical microphone angles based on the complete source integration volume with and without neglecting some of the mixed terms in the acoustic integral to investigate the effect of mutual orthogonality of individual source terms.

The total spectra solution and the reference experimental data are included in the same figures for comparison. The two approximate solutions shown correspond to (i) assuming the orthogonality of different azimuthal modes $\sum_{n} \overline{\hat{p}^{(n)}(\boldsymbol{x}, \omega) \hat{p}^{(n) *}(\boldsymbol{x}, \omega)}$ and (ii) further assuming the orthogonality of different source terms $\sum_{n} \overline{\hat{p}_{e_{4}}^{(n)}(\boldsymbol{x}, \omega) \hat{p}_{e_{4 j}}^{(n) *}(\boldsymbol{x}, \omega)}+$ $\sum_{n} \overline{\hat{p}_{\nabla_{j} \tilde{v}_{l}}^{(n)}(\boldsymbol{x}, \omega) \hat{p}_{\nabla_{j} \tilde{v}_{l}}^{(n) *}(\boldsymbol{x}, \omega)}+\sum_{n} \overline{\hat{p}_{e_{l \jmath}}^{(n)}(\boldsymbol{x}, \omega) \hat{p}_{e_{l \jmath}}^{(n) *}(\boldsymbol{x}, \omega)}$ for the far field sound power spectra in (17). Note that both the approximate solutions are within 1-2 dB from the total spectra for most frequencies which confirms validity the assumption of mutual orthogonality for different stress term components in the SILOET jet case.

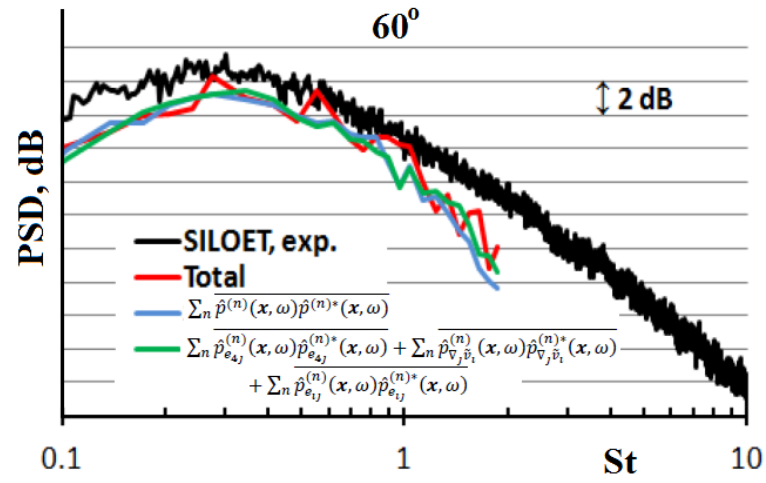

(a)

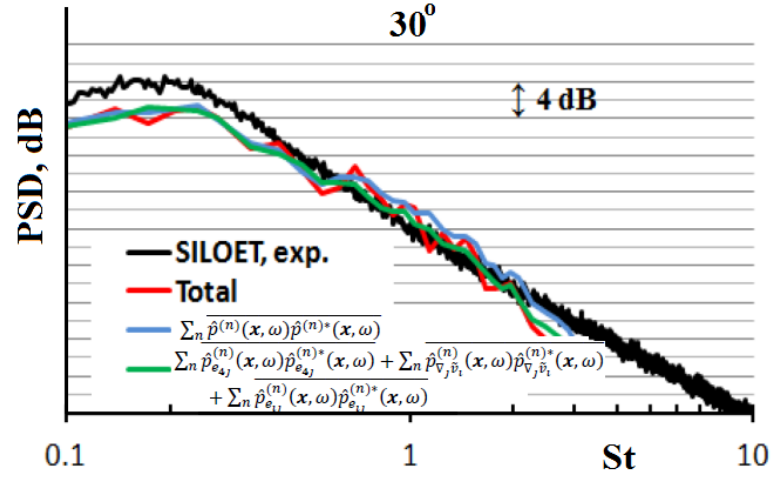

(b)

Fig.9 Orthogonality of individual source term components for far field sound spectra at $60^{\circ}$ (a) and $30^{\circ}$ angle (b) to the jet flow. The predictions shown correspond to the total spectra, the spectra solutions with assuming the orthogonality of different azimuthal modes of the source, and assuming the orthogonality of different stress terms. 


\subsection{Effect of the source type and directivity on far-field noise}

Fig.10 shows contributions to the far-field noise spectra produced by various turbulent fluctuating stresses in comparison with the total spectra. There are contributions of various components of the fluctuating Reynolds stresses, $e^{\prime \prime}{ }_{i j}$ considered (i,j=1,2,3), where $\boldsymbol{e}_{1}$ is the Cartesian coordinate in the jet flow direction, $\left(\boldsymbol{e}_{2}, \boldsymbol{e}_{3}\right)$ are in the transverse plane, and $\left(\boldsymbol{e}_{1}, \boldsymbol{e}_{2}\right)$ are in-plane with the far-field microphone as discussed in section 2.2. The contributions of the enthalpy fluctuation term, $\hat{e}^{\prime \prime}{ }_{4 j}(\boldsymbol{y}, \omega)$ and the term, which includes the velocity divergence $\hat{e}^{\prime \prime}{ }_{i j}(\boldsymbol{y}, \omega) \nabla_{j} \tilde{v}_{i}(\boldsymbol{y})$ are also shown. The integration domain corresponds to the entire jet source volume.

Fig.10a shows that for $90^{\circ}$ microphone angle the contribution of the enthalpy term and the velocity divergence term to the far-field noise is negligible compared to the fluctuating Reynolds stresses. This is the generalised stress term which corresponds to the Lighthill quadrupole noise source. Fluctuating Reynolds stresses remain the dominant noise component term for other polar angles as well. However, the directivity of the dominant Reynolds stress component changes depending on the polar angle. For example, the $e^{\prime \prime}{ }_{22}$ stress component is most important at $90^{\circ}$ angle, $\hat{e}^{\prime \prime}{ }_{22}, \hat{e}^{\prime \prime}{ }_{21}, \hat{e}^{\prime \prime}{ }_{12}, \hat{e}^{\prime \prime}{ }_{11}$ become all significant for $60^{\circ}$, and $\hat{e}^{\prime \prime}{ }_{11}, \hat{e}^{\prime \prime}{ }_{12}, \hat{e}^{\prime \prime}{ }_{21}$ are important for $30^{\circ}$. The relative contribution of the Reynolds stress components also depends on the sound frequency. For example, the relative importance of $e^{\prime \prime}{ }_{12}$ increases at high frequencies while $e^{\prime \prime}{ }_{21}$ is most important at low frequencies and small angles to the jet flows. The contribution of the out-of-plane components remains negligible for all angles and frequencies. 


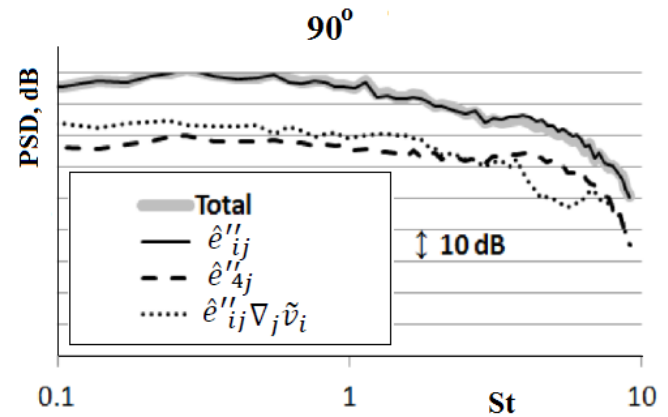

(a)

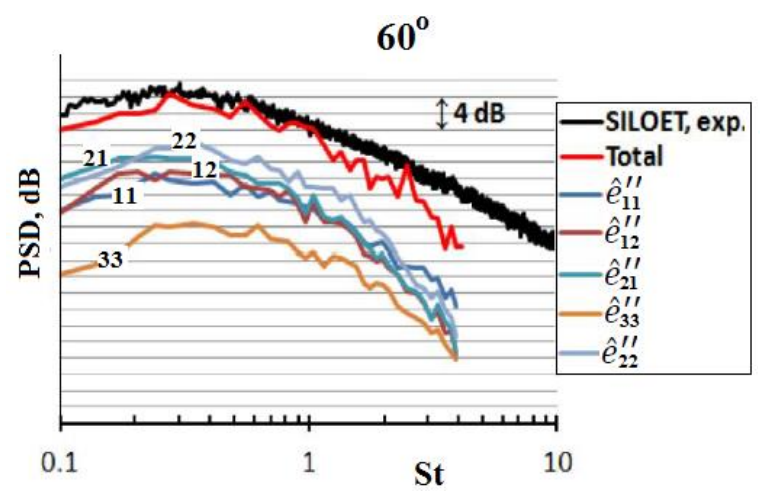

(c)

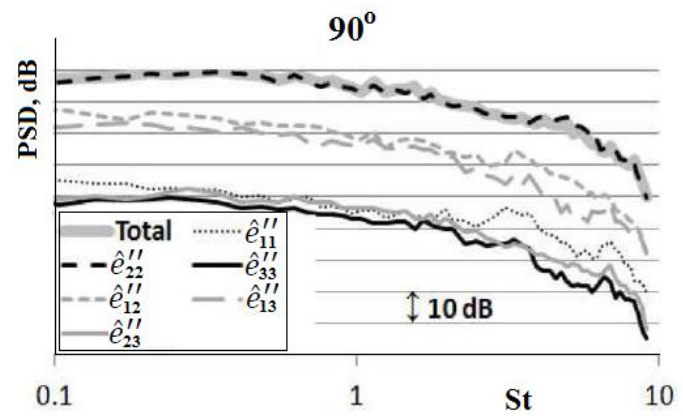

(b)

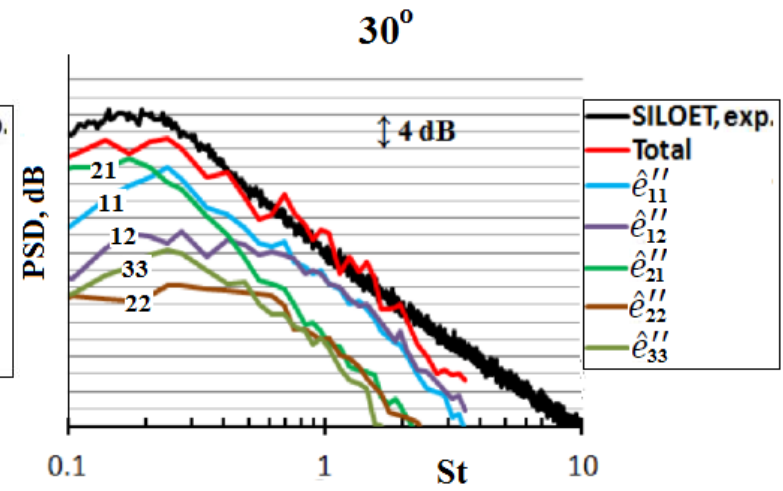

(d)

Fig.10 Contribution of individual fluctuating stresses to far-filed noise spectra: comparison of the fluctuating Reynolds stress term with the fluctuating enthalpy and the velocity divergence terms for $90^{\circ}$ polar angle (a), comparison of various components of the fluctuating Reynolds stress term for $90^{\circ}$ polar angle (b), comparison of various components of the fluctuating Reynolds stress term for $60^{\circ}$ polar angle (c), comparison of various components of the fluctuating Reynolds stress term for $30^{\circ}$ polar angle (d).

\subsection{Contribution of the azimuthal source modes to the far-field noise}

Fig.11 shows the contribution of different modes of the fluctuating Reynolds stresses to the far field noise spectra for different polar angles. The total spectra solution based on the same complete integration volume and the experiment data are shown on the same plots for comparison. 
For the $30^{\circ}$ angle to the jet flow, the axi-symmetric azimuthal mode, $n=0$ is the most dominant component in the frequency range $0.2<\mathrm{St}<0.8$, where St 0.2 corresponds to the lowest numerically frequencies, which are numerically captured for sound spectra predictions at this polar angle (see discussion in section 3). This observation is in agreement with the existing experiments (Cavalieri at al. 2013) which showed that it is the zero order azimuthal mode, $n=0$ which mostly contributes to jet noise at the $30^{\circ}$ polar angle.

For high polar angles, $60^{\circ}$ and $90^{\circ}$ to the jet flow, the first asymmetric azimuthal mode, $n=1$ becomes increasingly important starting from mid frequencies, $S t \geq 0.4$. As the sound frequency increases the importance of other high order modes also increases. The increase in relative importance of high order modes with the frequency is most notable at the $90^{\circ}$ polar angle compared to the small angles to the jet flow. For example, at $S t=1$ and $30^{\circ}$ polar angle there are only a few significant modes, $n=0$ and 1 that contribute to the far-field noise spectra efficiently, while for the $60^{\circ}$ polar angle it is the $n=1$ mode which is most significant, and for the $90^{\circ}$ angle these are the modes $n=1$ and 2, which contribute to the far-field noise effectively. All this is also consistent with the analysis presented in (Goldstein 1976) and (Goldstein et al. 2012) who showed that the $n=0$ mode is highly directional, hence, does not significantly contribute to the $90^{\circ}$ noise spectrum. Notably, the effect of high-order azimuthal modes on sound spectra at large polar angles to the jet flow, which is captured by the current calibration-free implementation of the generalised acoustic analogy, would be difficult to reproduce by approaches based on modelling of coherent large-scale structures that stem from the instability wave theory. 


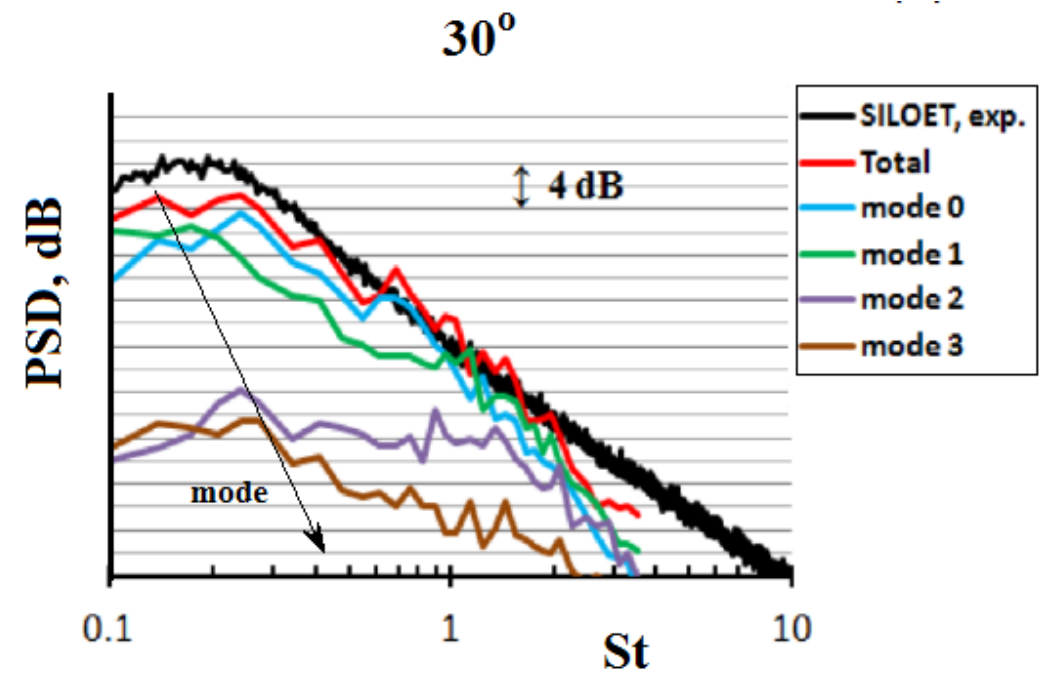

(a)

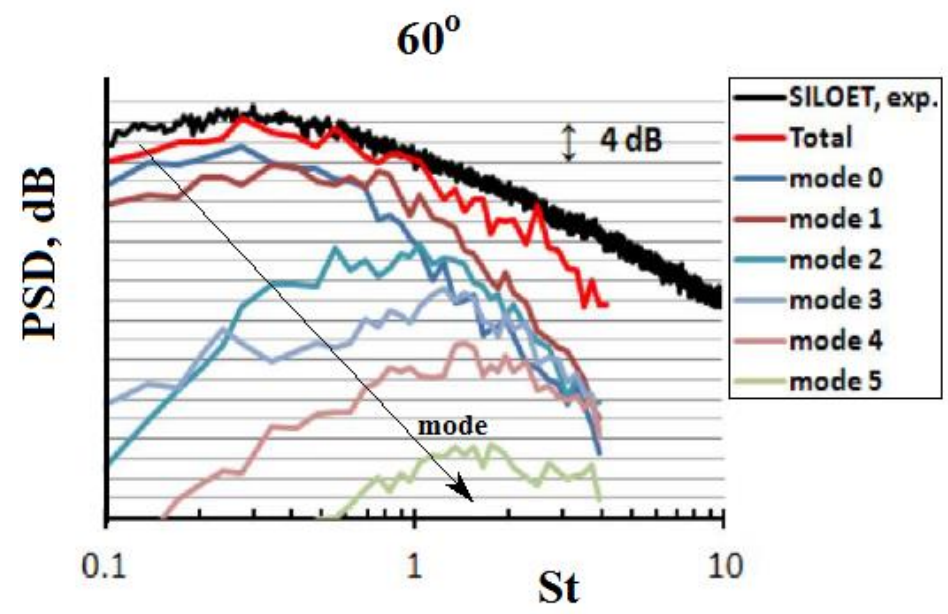

(b) 


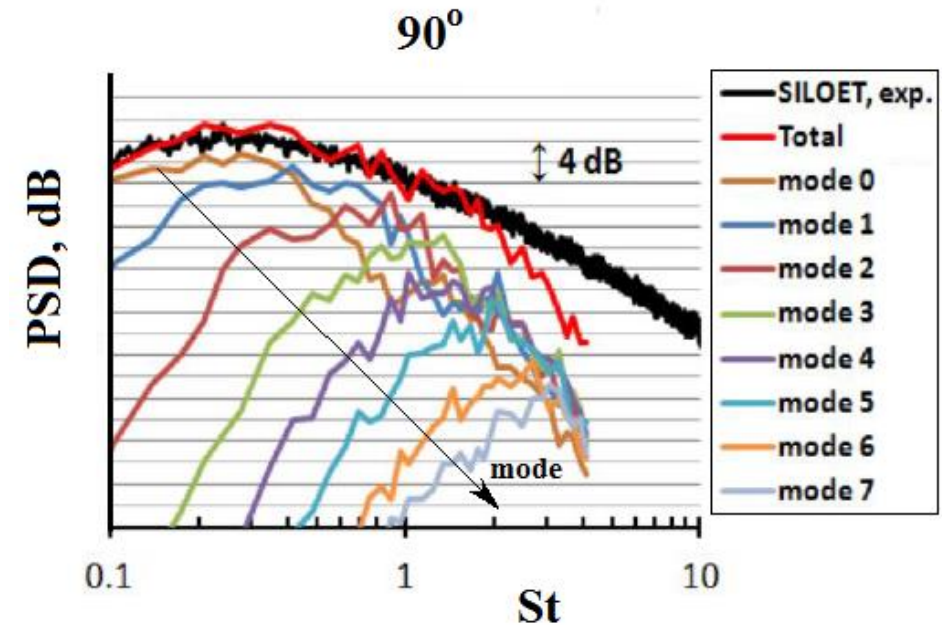

(c)

Fig. 11 Contribution to the far-field noise spectra from different azimuthal modes of the fluctuating Reynolds stresses for $30^{\circ}$ (a), $60^{\circ}(\mathrm{b})$, and $90^{\circ}$ (c) polar angle to the jet flow.

\subsection{Effect of the meanflow sound propagation on the far-field noise}

In addition to the effect of the source integration volume and individual source components, the effect of meanflow sound propagation is an important contributor to the far-field noise. In this sub-section, the effect of the Green's function on the far-field solution is analysed by comparing the noise predictions obtained with the locally parallel solution and those obtained with the free-space solution that completely ignores the jet refraction effect. In each case, the integration volume and the noise source description are fixed to be the same and correspond to a complete set of the fluctuating stresses, $e^{\prime \prime}{ }_{\mu j}$ of the Goldstein generalised acoustic analogy.

Both noise spectra solutions, with and without taking the meanflow effect into account, are integrated in the frequency band $0.05<S t<2$ and compared with the corresponding Over All Sound Pressure Level (OASPL) data from the SILOET experiment.

The results are shown in Table 3 which shows the differences between the reference OASPL data and the two far-field predictions of the volume integral method based on the Goldstein 
generalised acoustic analogy with and without considering the meanflow propagation effect as a function of the polar angle. The error for the solution which includes the corresponding Green's function that takes into account the meanflow effect remains within $0.5-2.2 \mathrm{~dB}$. Compared to this, the error of the same volume integral method based on the free-space Green's function monotonically grows with decrease of the polar angle and reaches $6 \mathrm{~dB}$ for $30^{\circ}$ microphone angle. Note that the error can be expected to be much larger at the higher Mach numbers corresponding to take off conditions. This error growth signifies the importance of meanflow propagation effects for small angles of noise radiation to the jet flow. The latter is in agreement with the exiting jet noise literature that discusses the effect of mean flow sound propagation modelling on noise spectra predictions at small angles to the jet flow (comp. with fig.16 from (Karabasov at al. 2010) and fig.12a,b from (Karabasov at al. 2013)).

For comparison, the same table also shows the OASPL error which corresponds to an integral volume implementation of the classical Lighthill acoustic analogy based on the LES solution of a high-speed jet case from (Bogey at al. 2001). For this case, there are no experimental data available and the reference "true solution" was defined by computing the LES solution inside a large control surface and then integrating the latter to the far-field using the Kirchoff method. Using the digitised data from (Bogey at al. 2001), the Lighthill acoustic analogy error is then specified as a difference between the reference "true solution" and the OASPL predictions of the Lighthill acoustic analogy solution. Note that the latter error fluctuates between $+2.5 \mathrm{~dB}$ and $-5 \mathrm{~dB}$ and does not show any particular trend with the polar angle. 
Table 3. Dependence of the OASPL error on the acoustic model and the polar angle.

\begin{tabular}{|l|l|l|l|}
\hline & OASPL error & OASPL error & OASPL error \\
& at $90^{\circ}, \mathrm{dB}$ & at $60^{\circ}, \mathrm{dB}$ & at $30^{\circ}, \mathrm{dB}$ \\
\hline Goldstein acoustic analogy with meanflow & 0.5 & 2 & 2.2 \\
\hline Sound propagation modelling & & & \\
\hline sound propagation modelling & 0.5 & 3.2 & 6 \\
\hline Lighthill acoustic analogy (Bogey et al. & 2.5 & -5 & 2.5 \\
\hline 2001) & & & \\
\hline
\end{tabular}

\section{Conclusions}

A new volume integral formulation of the Goldstein generalised acoustic analogy is developed. On the one hand, it can be seen as a significant extension of the previous volume integral methods in the acoustic analogy literature. On the other hand, the new method can be regarded as a new volume integral implementation of the generalised acoustic analogy, which does not require the calculation and recording of the expensive generalised stress tensor autocovariance function and is automatically extendable to asymmetric jets. It is also shown how the new implementation allows a direct correspondence between the fluctuating stress components in the jet flow and their effect for the far-field noise power spectra. Thanks to these properties, the new volume integral method can fully utilise the availability of timedependent, three-dimensional LES flow solutions to investigate jet noise mechanisms at a new level of detail compared to the state-of-the art experimental or theoretical studies. Examples of the modelling are provided for a particular choice of the LES method, isothermal subsonic jet, and jet inflow conditions but the general approach developed is 
suitable for any DNS, LES, or hybrid LES-Reynolds Averaged Navier-Stokes (RANS) techniques, and jet flow of the user choice. For example, the suggested volume integral method is sufficiently robust and requires only minor modifications for supersonic jet noise predictions, where an application of the critical layer correction in the locally parallel Green's function solution would be required (Goldstein and Leib 2008).

For validation of the new volume integral formulation, the high-speed subsonic isothermal static jet case corresponding to the Rolls-Royce SILOET experiment is considered. The SILOET jet flow shares many similarities with other high-speed jets in the literature both in terms of the flow characteristics and the turbulent velocity spectra. The SILOET flow solution is calculated with the Monotonically Integrated LES CABARET solver. For simplicity of the modelling, an idealised laminar condition is imposed at the nozzle exit, which choice is consistent with jet noise literature. The validation is performed using the reference experimental and computational data available and also the existing theory of Kolmogorov for turbulent velocity spectra. The far-field noise spectra predictions of the new volume integral method are validated against the far-field microphone data of the SILOET experiment with $2-3 \mathrm{~dB}$ agreement reported for a range of frequencies, $0.1<S t<2$ and polar angles, $60^{\circ}-90^{\circ}$ with respect to the jet flow. A similar agreement with the experiment is achieved for the same LES data when using the standard penetrable formulation of the Ffowcs Williams -Hawkings (FW-H) surface integral method.

For the $30^{\circ}$ angle to the jet flow and frequencies below $\mathrm{St}=0.2$, both the acoustic analogy and the FW-H solution under predict the experiment by $4 \mathrm{~dB}$, which is likely due to the insufficient LES grid resolution at the end of the potential core of the jet. For this polar angle, the noise spectrum prediction of the FW-H method appears to be more accurate $(0.5-1 \mathrm{~dB}$ error) in comparison with the acoustic analogy solution (2-3dB error). This difference can be explained by the approximate locally parallel Green's function solution used in the current 
acoustic analogy implementation as well as the opportunistic calibration involved in the selection of the optimal FW-H integration surface. Most importantly, compared to the volume integral method, the predictions of the surface integral method are sensitive to the control surface location and, furthermore, cannot be used to analyse the jet sources inside the jet.

To demonstrate capabilities of the new volume integral formulation of the Goldstein generalised acoustic analogy in revealing the salient properties of jet noise, the sound generation and propagation effects of the model are systematically analysed.

First, by truncating the integration volume in the radial and stream-wise directions, it is shown that the effective source correlation volume is proportional the physical jet dimensions. The effective source size tends to reduce with frequency without changing its shape. The high-frequency source region is located more compactly near the nozzle exit and the jet lipline compared to the low frequency noise sources, which are more distributed, in agreement with the existing jet noise experiments (Tam at al. 2008).

Then, it is shown that the individual stresses in terms of their modes and components are mutually orthogonal in the far-field spectra: the result of ignoring the mixed terms in the farfield noise spectra is virtually identical to the full noise spectra of the SILOET jet considered. This is in agreement with other publications in the jet noise literature that consider only symmetric terms of the generalised stress tensor auto-covariance, $R_{\mu j \mu j}$ to be important for jet noise and ignore the effect of different azimuthal mode coupling for axisymmetric jet flows. The effect of individual source types, such as the fluctuating Reynolds stress components, the enthalpy fluctuation term, and the velocity divergence term, and also that of different azimuthal source modes on the far-field noise spectra is analysed. Here, it is demonstrated that the fluctuating Reynolds stress is the dominant component for all polar angles compared to other source types. This is in agreement with the quadruple noise source behaviour expected in the isothermal jet noise case considered. Furthermore, it is shown how the 
relative importance of individual Reynolds stress changes depending on their directivity, the polar angle, and the sound frequency. In particular, the most efficient components of the fluctuating Reynolds stresses are always in-plane with the observer position. For $90^{\circ}$ polar angle, it is just one stress component that is important and which corresponds to the fluctuating stress pointing in the observer direction. For $60^{\circ}$ and $30^{\circ}$ angles to the jet, important noise sources have multiple directivity and include several longitudinal and transverse stress components. It also shown how the importance of high order azimuthal modes grows with sound frequency and the polar angle. In particular, in agreement with the experiments (Cavalieri at al. 2013) it is shown that it is the zero order azimuthal mode, $n=0$ which mostly contributes to jet noise at the $30^{0}$ polar angle to the jet flow. In comparison with this, the azimuthal modes $n=1$ and 2 are very important for sound radiation at $90^{\circ}$ angle, which is in agreement with the previous analysis (Goldstein 1975; Goldstein at al. 2012). Notably, the effect of the importance of high-order azimuthal modes for sound radiation at large polar angles to the jet flow, which has been captured here, would be difficult to reproduce by other methods such as those based on instability wave modelling of large-scale structures.

Finally, it is demonstrated that the meanflow propagation plays a key role for the far-field noise for small angles to the jet flow. The explicit accounting for the meanflow propagation effects is a distinctive feature of the Goldstein generalised acoustic analogy compared to other acoustic analogy models such as the Lighthill analogy. Compared to the generalised acoustic analogy, the Lighthill model can be prone to serious errors in overall sound pressure levels when implemented with LES that cannot fully resolve the entire range of acoustically important flow scales including both the turbulence and the meanflow propagation effects. As a final remark, it should be recalled that all sound predictions with the current volume integral method have been obtained using the simplified locally parallel flow model for sound 
propagation. While the incorporation of more complex meanflow propagation models, which would account for evolving jet effects, will be a subject of the future work, the 2-3dB accuracy of the current noise spectra predictions for most angles and frequencies calls for a revision of some earlier concepts and theories. For example, in a number of works in the literature on the Goldstein generalised acoustic analogy, there was a strong sensitivity of high-speed jet noise predictions to the evolving jet effects reported, hence, a high importance of these effects for jet noise predictions was concluded. But all these models were based on modelling the fluctuating stress auto-covariance function in the seven-dimensional spacetime and using some approximations for a convolution product of the source function with the Green's function to obtain the final noise spectra. Hence, there occurs a possibility that it was actually the exaggerated sensitivity of these models to the source model calibration parameters rather than details of the jet flow evolution, which was the real reason behind the previous conclusions. This calls for a new investigation of the importance of evolving jet effects for far-field noise spectra predictions based on the suggested calibration-free volume integral approach.

\section{Acknowledgement}

The work has been partially supported by the UK Engineering and Physical Sciences Research Council (EP/I017747/1) and partially by Aero Acoustics Research Consortium (AARC). The authors are grateful to the UK Government for supporting the SILOET program during which the model-scale data was acquired in the QinetiQ NTF and Dr Paul Strange (Rolls-Royce Plc) for facilitating access to these data. The authors are grateful to Dr Anton Markesteijn (GPU-Prime) for valuable discussions. 


\section{References}

1. Afsar, M.Z. Goldstein, M.E. and Fagan A. 2011 "Enthalpy-Flux/Momentum-Flux Coupling in the Acoustic Spectrum of Heated Jets", AIAA Journal, Vol. 49, No. 11 (2011), pp. 2522-2531.

2. Afsar, MZ, Sescu, A, Leib, SJ, 2016 "Predictive capability of low frequency jet noise using an asymptotic theory for the adjoint vector Green's function in non-parallel flow”, AIAA-2016-2804.

3. Bodony, D. and Lele, S. K. 2008 "Current status of jet noise predictions using largeeddy simulation.” AIAA J., 46:364-380.

4. Bogey, C., Bailly, C., Juve, D. 2001 “Noise computation using Lighthill's equation with inclusion of mean flow - acoustic interactions", AIAA paper 2001-2255.

5. Bogey C. and Bailly C. 2010 "Influence of nozzle-exit boundary-layer conditions on the flow and acoustic fields of initially laminar jets", J. Fluid Mech., Vol.663, 2010, 507-540.

6. Bogey, C., Marsden, O., and Bailly, C. 2012 "Influence of initial turbulence level on the flow and sound fields of a subsonic jet at a diameter-based Reynolds number of $10^{5}$, J. Fluid Mech. Volume 701, pp. 352-385.

7. Brentner, K. S. and Farassat, F. 1998 "Analytical Comparison of the Acoustic Analogy and Kirchhoff Formulation for Moving Surfaces," AIAA Journal, Vol. 36, No. 8, pp. 1379-1386.

8. Bres, G.A., Nichols, J.A., Lele, S.K., Ham, F.E., Schlinker, R.H., Reba, R.A. and Simonich, J.C. 2012 “Unstructured Large Eddy Simulation of a Hot Supersonic OverExpanded Jet with Chevrons", 18th AIAA/CEAS Aeroacoustics Conference (33rd AIAA Aeroacoustics Conference), AIAA Paper 2012-2213. 
9. Bres, G.A., Jaunet, V., Le Rallic, M., Jordan, P., Colonius, T., and Lele, S.K. 2015 "LES for jet noise: the importance of getting the boundary layer right", AIAA Paper 2015-2535.

10. Bridges, J. and Wernet, M. 2003 "Measurements of Aeroacoustic Sound Sources in Turbulent Jets", AIAA paper 2003-3130.

11. Bridges, J. 2010 "Establishing Consensus Turbulence Statistics for Hot Subsonic Jets", AIAA paper 2010-3751.

12. Cavalieri, A.V.G., Rodriguez, D., Jordan. P., Colonius, T. \& Gervais, Y. 2013, "Wavepackets in the Velocity Field of Turbulent Jets," Journal of Fluid Mechanics, Vol.730, pp. 559-592.

13. Chintagunta, A., Naghibi, S.E., and Karabasov, S.A. 2017 "Flux-corrected dispersionimproved CABARET schemes for linear and nonlinear wave propagation problems", Computers \& Fluids, https://doi.org/10.1016/j.compfluid.2017.08.018.

14. Curle, N., 1955 “The Influence of Solid Boundaries upon Aerodynamic Sound”, DOI: $10.1098 /$ rspa. 1955

15. Depuru Mohan, N.K., Dowling, A.P., Karabasov, S.A., Xia, H. Graham, O., Hynes, T.P. and Tucker, P.G. 2015 “Acoustic Sources and Far-field Noise of Chevron and Round Jets", AIAA Journal, 30 Jun 2015, pp. 1-16.

16. Faranosov, G. A., Goloviznin, V. M., Karabasov, S. A., Kondakov, V. G., Kopiev,V. F., Zaitsev, M. A. 2013 “CABARET method on unstructured hexahedral grids for jet noise computation Computers and Fluids", 88, 165-179.

17. Ffowcs Williams, J. E. 1963 "The noise from turbulence convected at high speed". Phil Trans Roy Soc. Lond. 255, 469-503.

18. Ffowcs Williams, J. E., Hawkings, D. L. 1969 "Sound generation by turbulence and surfaces in arbitrary motion”, Philos. Trans. R. Soc., A264, 32142. 
19. di Francescantonio, P. 1997 "A New Boundary Integral Formulation for the Prediction of Sound Radiation," Journal of Sound and Vibration, Vol. 202, No. 4, pp. 491-509.

20. Freund, J. B. 2003 "Noise-source turbulence statistics and the noise from a Mach 0.9 jet Physics of Fluids", 15(6), 1788-1799.

21. Fureby, C., and Grinstein. F. F. 2002 "Large Eddy Simulation of High-ReynoldsNumber Free and Wall-Bounded Flows", Journal of Computational Physics, 181, 6897.

22. Goldstein, M. E., 1975, “The Low Frequency Sound from Multipole Sources in Axisymmetric Shear Flows, with Application to Jet Noise", Journal of Fluid Mechanics, Vol. 70, Part 3, pp. 595-604.

23. Goldstein, M. E. 2002 "A unified approach to some recent developments in jet noise theory" Int. Journ. Aeroacoustics, 1(1), 1-16.

24. Goldstein, M. E. 2003 “A generalized acoustic analogy” J. Fluid Mech., 488, 315333.

25. Goldstein, M. E. and Leib, S. J. 2008 "The Aero-acoustics of slowly diverging supersonic jets” J. Fluid Mech., 600, 291-337.

26. Goldstein M.E. 2010 “Relation between the generalized acoustic analogy and Lilley’s contributions to aeroacoustics", Int. J. Aeroacoustics, volume 9, number $4 \& 5$, pp. $401-418$.

27. Goldstein M.E. 2011 "Recent developments in the application of the Generalized Acoustic Analogy to jet noise prediction", Int. J. Aeroacoustics, volume 10, number 2\&3, pp. 89-116. 
28. Goldstein, M.E., Sescu, A., and Afsar M.Z. 2012 “Effect of non-parallel mean flow on the Green's function for predicting the low-frequency sound from turbulent air jets”, J. Fluid.Mech. 2012/3, 695, pp. 199-234.

29. Goldstein, M.E. and Leib, S.J. 2016 “Azimuthal Source Non-Compactness and Mode Coupling in Sound Radiation from High-Speed Axisymmetric Jets", AIAA Paper 2016-2803.

30. Goloviznin, V.M., Samarskii, A.A. 1998 "Finite difference approximation of convective transport equation with space splitting time derivative”. J. Matem. Mod. 10(1), 86-100.

31. Hussein, H.J., Capp, S.P. and George, W.K. 1994 "Velocity measurements in a highReynolds-number, momentum conserving, axisymmetric, turbulent jet.” J. Fluid Mech., 258:31-75.

32. Ingraham, D. and Bridges, J. E. 2017 "Validating a Monotonically-Integrated Large Eddy Simulation Code for Subsonic Jet Acoustics”, AIAA Paper 2017-0456.

33. Karabasov, S. A. and Hynes, T. P. 2006 "Adjoint Linearized Euler solver in the frequency domain for jet noise modelling. AIAA Paper 2006-2673.

34. Karabasov, S. A., and Goloviznin, V. M. 2009 "Compact Accurately Boundary Adjusting high-REsolution Technique for Fluid Dynamics” J. Comput. Phys., 228, 7426-7451.

35. Karabasov, S. A., Afsar, M. Z., Hynes, T. P., Dowling, A. P., McMullan, W. A., Prokora, C. D., Page, G. J. and McGuirk, J. J. 2010 "Jet Noise: Acoustic Analogy informed by Large Eddy Simulation”, AIAA Journal , 48(7), 1312-1325.

36. Karabasov, S. A. 2010 "Understanding Jet noise”, Phil. Trans. R. Soc. A, 368, 35933608. 
37. Karabasov, S. A., Bogey, C. and Hynes, T. P. 2013 "An investigation of the mechanisms of sound generation in initially laminar, subsonic jets using the Goldstein acoustic analogy”, J.Fluid Mech., 714, 24-57.

38. Karabasov S.A. and Sandberg R.D. 2015 "Influence of free stream effects on jet noise generation and propagation within the Goldstein acoustic analogy approach for fully turbulent jet inflow boundary conditions". Int. J. Aeroacoustics vol. 14, (3-4) 413430.

39. Lau, J. C, Morris, P. J, Fisher, M. J. 1979 "Measurements in subsonic and supersonic free jets using a laser velocimeter" J. Fluid Mech, 93(01), 1-27.

40. Leib, S. J. and Goldstein, M. E. 2011 "Hybrid Source Model for Predicting HighSpeed Jet Noise” AIAA Journal , 49(7), 1324-1335.

41. Leib, S.J., Ingraham, D., and Bridges, J.E. 2017 "Evaluating Source Terms of the Generalized Acoustic Analogy using the Jet Engine Noise REduction (JENRE) Code", AIAA Paper 2017-0459.

42. Lilley, G.M. 1958 “On the Noise from air jets.” Aeronaut. Res. Council Rep. Mem. 20,376 .

43. Lighthill, M. J. 1952 "On Sound Generated Aerodynamically: I. General Theory" Proceedings of the Royal Society of London, 546-587.

44. Markesteijn, A.P. and Karabasov, S.A. "CABARET solutions on Graphics Processing Units for NASA jets: grid sensitivity and unsteady inflow condition effect", CR Mecanique (Proc. French Academy of Sciences), in press.

45. Morris, P.J. Zaman, K.B.M.Q. "Velocity measurements in jets with application to noise source modeling”, J Sound and Vibration 329 (2010), 394-414.

46. Najafi-Yazdi, A., Bres, G. A., and Mongeau, L. 2011 "An acoustic analogy formulation for moving sources in uniformly moving media Proceedings of The 
Royal Society A Mathematical Physical and Engineering Sciences”, 467(2125), pp. 144-165.

47. Pope, S. B. 2000 “Turbulent flows Cambridge University Press”, New York.

48. Samanta, A., Freund, J. B., Wei, M. and Lele, S. K. 2006 "Robustness of acoustic analogies for predicting mixing-layer noise.” AIAA J. 44, 2780-2786.

49. Sassanis, S., Afsar, M., Sescu, A., Lele, L. 2017 "On the existence of an overlap region between the Green's function for a locally parallel axi-symmetric jet and the leading order non-parallel flow solution", 70th Annual Meeting of the APS Division of Fluid Dynamics, Volume 62, Number 14.

50. Shur, M. L., Spalart, P. R., Strelets, M. Kh. 2005 "Noise Prediction for increasingly complex jets. Part I: Methods and tests. Part II: Applications Int. J. Aeroacoustics.”, 4(34), 21366.

51. Shur, M. L., Spalart, P. R., Strelets, M. Kh., Travin, K. T. 2015 “An Enhanced Version of DES with Rapid Transition from RANS to LES in Separated Flows", Turbulence Flow Combust, 95(4), 709737.

52. Semiletov, V. A., and Karabasov, S. A. 2013 "CABARET scheme with conservationflux asynchronous time-stepping for nonlinear aeroacoustics problems." J. Comp. Physics, 253(15), 157165.

53. Semiletov, V. A., Karabasov, S. A. 2014 "CABARET scheme for computational aero acoustics: extension to asynchronous time stepping and 3D flow modelling", Int. J Aeroacoustics , 13(3,4), 321-336.

54. Semiletov, V. and Karabasov, S. 2014 "Adjoint Linearised Euler solver for Goldstein acoustic analogy equations for 3D non-uniform flow sound scattering problems: verification and capability study", AIAA Paper 2014-2318. 
55. Semiletov, V.A., Karabasov, S.A., Chintagunta, A. and Markesteijn, A.P. 2015 "Empiricism-free noise calculation from LES solution based on Goldstein generalized acoustic analogy: volume noise sources and meanflow effects", AIAA paper 20152536.

56. Semiletov, V.A., Karabasov S.A. 2017 "On the similarity scaling of jet noise sources for low-order jet noise modelling based on the Goldstein generalised acoustic analogy”, International Journal of Aeroacoustics, Volume: 16 issue: 6, 476-490.

57. SILOET Programme Rolls-Royce private data.

58. Tam, C. K. W. and Auriault, L. 1998 "Mean flow refraction effects on sound from localized sources in a jet”, J. Fluid Mech., 370, 149-174.

59. Tam, C.K.W., Viswanathan, K., Ahuja, K.K. and Panda, J. 2008 "The sources of jet noise: experimental evidence J. Fluid Mech., 615, 253-992.

60. Viswanathan, K. 2004 “Aeroacoustics of hot jets”, J. Fluid Mech. 516, pp. 39-82.

61. Viswanathan, K. 2009 "Mechanisms of jet noise generation: classical theories and recent developments", Intl J. Aeroacoust. , 615, 253-992.

62. Welch, P. D. 1967 "The Use of Fast Fourier Transform for the Estimation of Power Spectra: A Method Based on Time Averaging Over Short, Modified Periodograms”, IEEE Transactions on Audio Electroacoustics , AU-15, 70-73.

63. Witze, P. O. 1974 "Centerline velocity decay of compressible free jets", AIAA J., 12(4), 417-418. 